\title{
Reclassification of the Genus Pasteurella Trevisan 1887 on the Basis of Deoxyribonucleic Acid Homology, with Proposals for the New Species Pasteurella dagmatis, Pasteurella canis, Pasteurella stomatis, Pasteurella anatis, and Pasteurella langaa
}

\author{
R. MUTTERS,${ }^{1 *}$ P. IHM, ${ }^{2}$ S. POHL,${ }^{3}$ W. FREDERIKSEN,${ }^{4}$ AND W. MANNHEIM ${ }^{1}$
}

Zentrum für Hygiene und Medizinische Mikrobiologie Klinikum der Universität, Department of Bacteriology, Pilgrimstein

2, D-3550 Marburg, Federal Republic of Germany ; Institut für Medizinisch-Biologische Statistik und Dokumentation der Universität, D-3550 Marburg, Federal Republic of Germany ${ }^{2}$; Hygiene-Institut der Universität, D-6900 Heidelberg, Federal Republic of Germany ${ }^{3}$; and Statens Seruminstitut, Diagnoseafdelingen, DK-2300 Copenhagen $S$, Denmark ${ }^{4}$

\begin{abstract}
Deoxyribonucleic acid (DNA)-DNA hybridization was used to determine the genetic relationships among a variety of previously established or proposed species of Pasteurella and their positions within the family Pasteurellaceae Pohl 1981. Our results indicated that the genus Pasteurella sensu stricto, which can be separated from the Actinobacillus group, consists of at least the following 11 species: Pasteurella multocida, with three subspecies ( $P$. multocida subsp. multocida, $P$. multocida subsp. septica, and $P$. multocida subsp. gallicida); Pasteurella dagmatis sp. nov., containing organisms previously labeled Pasteurella "gas," Pasteurella new species 1, or Pasteurella pneumotropica type Henriksen; Pasteurella gallinarum; Pasteurella canis sp. nov., previously labeled $P$. multocida biotype 6 or "dog type" strains; Pasteurella stomatis sp. nov., which contains Pasteurella strains isolated from dogs and cats; Pasteurella avium (Hinz and Kunjara) Mutters et al. 1985; Pasteurella volantium Mutters et al. 1985, a new species consisting of V-factor-requiring strains that occur in humans and birds; Pasteurella anatis sp. nov.; Pasteurella langaa sp. nov., containing strains previously designated taxa 1 and 4 of Bisgaard; and two new species, which were provisionally designated Pasteurella species A and Pasteurella species B. The previously recognized taxa Pasteurella ureae, Pasteurella haemolytica biotypes A and T, Pasteurella testudinis, and P. pneumotropica biotypes Jawetz and Heyl do not belong to the genus Pasteurella but are more closely related to the Actinobacillus group. The exact taxonomic positions of Pasteurella aerogenes, P. multocida biotype 1, group HB-5, "Pasteurella piscicida," the SP group, and the bovine lymphangitis group are still unknown, but these organisms do not belong to the genus Pasteurella.
\end{abstract}

Around 1880 certain microorganisms were described as probably associated with fowl cholera and hemorrhagic septicemia of cattle and other animals. In 1885 Kitt cultured the etiological agent (31). Not until around 1920 were similar or identical organisms reliably isolated and described from infections in humans $(51,63)$.

"Micrococcus gallicidus" Burrill 1883 (5) appears to be the first scientific name used for these bacteria. Later, this organism was assigned to various genera, including "Bacterium" (11, 31, 33, 34), "Octopsis" (65), "Coccobacillus" (14), and "Eucystia" (10). In 1887, Trevisan proposed the generic name Pasteurella to commemorate the work of Pasteur on these bacteria (66).

During the years that followed, many organisms with a variety of species names were assigned to the genus Pasteurella, mainly on the basis of bipolar staining or the host species from which the microorganism was isolated (26). When this fashion of naming species after their animal hosts (35) ended, the epithet septica, which was proposed by Topley and Wilson in 1929 (64), was adopted for Pasteurella in the Anglo-Saxon parts of the world. In 1939, Rosenbusch and Merchant used the epithet multocida (53), which had been used first by Kitt in 1893 in the combination "Bacterium bipolare multocidum' (32) and by Lehmann and Neumann in 1899 in the name Bacterium multocidium (34). Although the epithet gallicida, which was used by Burrill in 1883 (5), appears to have priority, Pasteurella multocida was proposed to be conserved as the most appropriate name (58).

\footnotetext{
* Corresponding author.
}

Until 1932, the genus Pasteurella consisted only of the type species, Pasteurella multocida. During the next 42 years the genus was expanded to include Pasteurella haemolytica by Newsom and Cross (48), Pasteurella pneumotropica by Jawetz (27), Pasteurella gallinarum by Hall et al. (19), Pasteurella ureae by Jones (29), and Pasteurella aerogenes by McAllister and Carter (43). Recently, many newly isolated microorganisms have been assigned to the genus Pasteurella, including Pasteurella "gas" or Pasteurella new species 1 (17), the SP group (13), the bovine lymphanggitis group (28), a bacterium with the Centers for Disease Control $\alpha$-numeric designation group HB-5 ("Pasteurella bettii"), and Pasteurella testudinis (61), as well as some new taxa isolated from poultry, guinea pigs, and calves $(1,2,35 a)$. With many of these new organisms, however, the difficulties of discriminating between members of the genus Pasteurella and members of the genus Actinobacillus Brumpt 1910 were significant.

Many investigators have considered the problems of phenotypic differentiation between these two genera. For example, in 1969 Mráz proposed reclassification of $P$. haemolytica as "Actinobacillus haemolyticus" because of the phenotypic similarity of this organism to the genus Actinobacillus (44). In 1973 Frederiksen suggested that $P$. ureae should be recognized as a species of the genus Actinobacillus because of its similarity to this group (12), a proposal that was in accordance with previous statements of Jones (29). In 1978 workers from American Type Culture Collection presented a phenotypic comparison of Pasteurella and Actinobacillus strains and stated that the two genera could not be separated 
TABLE 1. Strains used in this study

\begin{tabular}{|c|c|c|c|c|c|}
\hline $\begin{array}{l}\text { Laboratory } \\
\text { no. }\end{array}$ & Strain $^{a}$ & Taxon & Derivation and other information & Reference(s) & $\begin{array}{l}\mathrm{G}+\mathrm{C} \text { content } \\
(\mathrm{mol} \%)^{h}\end{array}$ \\
\hline 1 & NCTC $10322^{\mathrm{T}}$ & $\begin{array}{l}\text { P. multocida subsp. } \\
\text { multocida }\end{array}$ & $\begin{array}{l}\text { G. R. Carter strain W-9217; Carter serogroup A; } \\
\text { Frederiksen biotype 4; porcine. Canada }\end{array}$ & 54 & 41.6 \\
\hline 2 & IPDH $108-78$ & $\begin{array}{l}\text { P. multocida subsp. } \\
\text { multocida }\end{array}$ & K.-H. Hinz; chicken, Federal Republic of Germany & & 43.2 \\
\hline 3 & SSI P325 & $\begin{array}{l}\text { P. multocida subsp. } \\
\text { multocida }\end{array}$ & $\begin{array}{l}\text { W. Frederiksen strain 7566-64; Frederiksen biotype } \\
2 \text {; human }\end{array}$ & 12 & 42.8 \\
\hline 4 & SSI P296 & $\begin{array}{l}\text { P. multocida subsp. } \\
\text { multocida }\end{array}$ & $\begin{array}{l}\text { J. E. Smith strain } 711 \text {; human, dog bite, United } \\
\text { Kingdom }\end{array}$ & 55 & 42.2 \\
\hline 5 & HIM 709-4 & $\begin{array}{l}\text { P. multocida subsp. } \\
\text { multocida }\end{array}$ & $\begin{array}{l}\text { H. Gerlach strain 1449; ducklings, Federal Republic } \\
\text { of Germany }\end{array}$ & & 42.3 \\
\hline 6 & IPDH 630-78 & $\begin{array}{l}\text { P. multocida subsp. } \\
\text { multocida }\end{array}$ & K.-H. Hinz; duck, Federal Republic of Germany & & 43.9 \\
\hline 7 & NCTC 10323 & $\begin{array}{l}\text { P. multocida subsp. } \\
\text { multocida }\end{array}$ & $\begin{array}{l}\text { W. Frederiksen strain SSI P428; J. A. McKiel, } \\
\text { strain insein; Frederiksen biotype 5; Carter } \\
\text { serogroup 8; bovine, Burma }\end{array}$ & 3,12 & 43.0 \\
\hline 8 & NCTC 8080 & $\begin{array}{l}\text { P. multocida subsp. } \\
\text { multocida }\end{array}$ & $\begin{array}{l}\text { J. E. Smith strain 170; E. D. Hoare strain H; } \\
\text { human, United Kingdom }\end{array}$ & 55 & 42.1 \\
\hline 9 & NCTC 3195 & $\begin{array}{l}\text { P. multocida subsp. } \\
\text { multocida }\end{array}$ & H. Schütze strain HS; bovine, United Kingdom & & 40.8 \\
\hline 10 & HIM 729-5 & $\begin{array}{l}\text { P. multocida subsp. } \\
\text { multocida }\end{array}$ & R. C. Meyer strain $7 / 72$; United States & & 43.5 \\
\hline 11 & NCTC 10201 & $\begin{array}{l}P . \text { multocida subsp. } \\
\text { multocida }\end{array}$ & $\begin{array}{l}\text { W. Frederiksen strain SSI P424; R. S. Roberts } \\
\text { strain A4; Frederiksen biotype 3; Roberts } \\
\text { serotype II; avian, United Kingdom }\end{array}$ & 12,52 & 41.9 \\
\hline 12 & CIP A $125^{\mathrm{T}}$ & $\begin{array}{l}\text { P. multocida subsp. } \\
\text { septica }\end{array}$ & G. Girard strain Souchard; human, cat bite, France & & 41.5 \\
\hline 13 & NCTC 11619 & $\begin{array}{l}\text { P. multocida subsp. } \\
\text { septica }\end{array}$ & $\begin{array}{l}\text { Marburg strain 118100/81; human, Federal Republic } \\
\text { of Germany }\end{array}$ & & 42.5 \\
\hline 14 & NCTC 11620 & $\begin{array}{l}\text { P. multocida subsp. } \\
\text { septica }\end{array}$ & $\begin{array}{l}\text { H. Gerlach strain } 1425 \text {; budgerigar, Federal } \\
\text { Republic of Germany }\end{array}$ & & 42.5 \\
\hline 15 & HIM 551-1 & $\begin{array}{l}\text { P. multocida subsp. } \\
\text { septica }\end{array}$ & $\begin{array}{l}\text { Marburg strain 142910/76; human, dog bite, Federal } \\
\text { Republic of Germany }\end{array}$ & 25 & 43.5 \\
\hline 16 & NCTC $10204^{\mathrm{T}}$ & $\begin{array}{l}\text { P. multocida subsp. } \\
\text { gallicida }\end{array}$ & $\begin{array}{l}\text { W. Frederiksen strain SSI P426; R. S. Roberts } \\
\text { strain IV-739; bovine, United Kingdom }\end{array}$ & 52 & 41.2 \\
\hline 17 & SSI P697 & $\begin{array}{l}\text { P. multocida subsp. } \\
\text { gallicida }\end{array}$ & $\begin{array}{l}\text { W. Frederiksen; Steigerwalt strain CDC } 7987 \text {; } \\
\text { United States }\end{array}$ & & 41.9 \\
\hline 18 & SSI P808 & $\begin{array}{l}\text { P. multocida subsp. } \\
\text { gallicida }\end{array}$ & W. Frederiksen; Thorshavn strain FsK. 10752(83) & & 42.5 \\
\hline 19 & NCTC $11617^{\mathrm{T}}$ & P. dagmatis & S. D. Henriksen strain $953 / 60$; human, Norway & 23 & 40.0 \\
\hline 20 & NCTC 11618 & P. dagmatis & $\begin{array}{l}\text { W. Frederiksen; R. E. Weaver strain C } 9882 \text {; } \\
\text { human, dog bite, United States }\end{array}$ & 13 & 42.0 \\
\hline 21 & HIM $815-8$ & P. dagmatis & J. E. Smith strain 151 ; dog, United Kingdom & 56 & 39.4 \\
\hline 22 & SSI P497 & P. dagmatis & $\begin{array}{l}\text { W. Frederiksen; J. Haagsma strain } 2 / 68852 \text {; cat, } \\
\text { The Netherlands }\end{array}$ & 13,18 & 41.5 \\
\hline 23 & SSI P150 & P. dagmatis & W. Frederiksen; human, cat bite, Denmark & 13 & 41.9 \\
\hline 24 & HIM 661-8 & P. dagmatis & J. E. Smith strain $180 ;$ dog, United Kingdom & 56 & 38.9 \\
\hline 25 & ATCC $13361^{\mathrm{T}}$ & $P$. gallinarum & $\begin{array}{l}\text { K. L. Heddleston strain USDA P-913; chicken, } \\
\text { United States }\end{array}$ & 19,54 & 43.6 \\
\hline 26 & ATCC 13360 & P. gallinarum & $\begin{array}{l}\text { K. L. Heddleston strain USDA P-982; chicken, } \\
\text { United States }\end{array}$ & 19 & 43.7 \\
\hline 27 & NCTC $11621^{\mathrm{T}}$ & P. canis & $\begin{array}{l}\text { J. E. Smith strain } 182 \text {; Frederiksen biotype } 6 \text {; dog, } \\
\text { United Kingdom }\end{array}$ & $12,57,62$ & 37.8 \\
\hline 28 & NCTC 11622 & P. canis & $\begin{array}{l}\text { Marburg strain 40930/67; human, Federal Republic } \\
\text { of Germany }\end{array}$ & 25 & 37.7 \\
\hline 29 & HIM $712-4$ & P. canis & $\begin{array}{l}\text { Marburg strain 078805-I/81; human, Federal } \\
\text { Republic of Germany }\end{array}$ & & 39.8 \\
\hline 30 & HIM $651-5$ & P. canis & $\begin{array}{l}\text { S. D. Henriksen strain } 12505 / 73 \text {; Frederiksen } \\
\text { biotype } 6 \text {; dog, Norway }\end{array}$ & 21,62 & 39.3 \\
\hline 31 & HIM 843-5 & P. canis & $\begin{array}{l}\text { M. Bisgaard strain } \mathrm{K} 267 \text {; taxon } 13\left(\mathrm{Orn}^{+}\right) \text {; calf, } \\
\text { Denmark }\end{array}$ & $35 \mathrm{a}$ & 41.9 \\
\hline 32 & NCTC $11623^{\mathrm{T}}$ & P. stomatis & J. E. Smith strain 166; dog, United Kingdom & 56 & 42.2 \\
\hline 33 & NCTC 11624 & P. stomatis & $\begin{array}{l}\text { W. Mannheim strain PE4; cat, Federal Republic of } \\
\text { Germany }\end{array}$ & & 40.4 \\
\hline 34 & SSI P294 & P. stomatis & $\begin{array}{l}\text { J. E. Smith strain } 221 \text {; Frederiksen biotype 6; dog, } \\
\text { United Kingdom }\end{array}$ & 56 & 43.5 \\
\hline 35 & HIM $748-5$ & P. stomatis & J. E. Smith strain 150 ; dog, United Kingdom & 56 & 41.4 \\
\hline 36 & NCTC $11413^{\mathrm{T}}$ & $P$. anatis & M. Bisgaard strain F149; taxon 1; duck, Denmark & 1 & 39.9 \\
\hline 37 & HIM 745-4 & P. anatis & M. Bisgaard strain F279; taxon 1; duck, Denmark & 1 & 42.3 \\
\hline
\end{tabular}


TABLE $1-$ Continued

\begin{tabular}{|c|c|c|c|c|c|}
\hline $\begin{array}{l}\text { Laboratory } \\
\text { no. }\end{array}$ & Strain $^{a}$ & Taxon & Derivation and other information & Reference(s) & $\begin{array}{l}\mathrm{G}+\mathrm{C} \text { content } \\
\quad(\mathrm{mol} \%)^{b}\end{array}$ \\
\hline 38 & SSI P683 & Pasteurella species B & $\begin{array}{l}\text { V. Janeckova strain Aldova 23193; Frederiksen } \\
\text { biotype 6; cat, Czechoslovakia }\end{array}$ & & 40.0 \\
\hline 39 & SSI P809 & Pasteurella species B & $\begin{array}{l}\text { W. Frederiksen; Odense strain FsK. 11478; human, } \\
\text { dog bite }\end{array}$ & & 38.9 \\
\hline 40 & NCTC $1141^{\mathrm{T}}$ & P. langaa & M. Bisgaard strain F73; taxon 4; chicken, Denmark & 1 & 43.9 \\
\hline 41 & HIM $745-1$ & $P$. langaa & M. Bisgaard strain F74; taxon 4; chicken, Denmark & 1 & 45.3 \\
\hline 42 & ATCC $29546^{\mathrm{T}}$ & P. avium & $\begin{array}{l}\text { K.-H. Hinz strain IPDH 2654; chicken. United } \\
\text { States }\end{array}$ & $24,46,54$ & 44.7 \\
\hline 43 & IPDH 0002 & P. avium & $\begin{array}{l}\text { K.-H. Hinz strain TS9; chicken, Federal Republic } \\
\text { of Germany }\end{array}$ & 24,46 & 44.3 \\
\hline 44 & HIM 844-2 & P. avium & $\begin{array}{l}\text { M. Bisgaard strain K } 117 \text {; taxon } 13\left(\mathrm{Orn}^{-}\right) \text {; calf, } \\
\text { Denmark, }\end{array}$ & $35 \mathrm{a}, 46$ & 42.8 \\
\hline 45 & NCTC $3438^{\mathrm{T}}$ & P. volantium & R. Lovell strain 6; fowl, United Kingdom & 24,46 & 43.8 \\
\hline 46 & NCTC 4101 & P. volantium & A. Fleming strain 1; human, United Kingdom & 46 & 44.6 \\
\hline 47 & IPDH 0003 & $P$. volantium & $\begin{array}{l}\text { K.-H. Hinz strain TS8; chicken. Federal Republic } \\
\text { of Germany }\end{array}$ & 24,46 & 44.7 \\
\hline 48 & $\operatorname{ATCC} 27883^{\mathrm{T}}$ & P. aerogenes & $\begin{array}{l}\text { H. A. McAllister strain P-172-71; swine, United } \\
\text { States }\end{array}$ & 43,54 & 41.8 \\
\hline 49 & HIM 612-4/5 & P. aerogenes & G. R. Carter strain SS-274-74; swine. United States & 13,43 & 43.1 \\
\hline 50 & NCTC 10535 & "P. bettii" & $\begin{array}{l}\text { D. G. Hollis strain CDC 41-5568; Centers for } \\
\text { Disease Control group HB-5; human. United } \\
\text { States }\end{array}$ & & 38.6 \\
\hline 51 & NCTC $9380^{\mathrm{T}}$ & P. haemolytica- & $\begin{array}{l}\text { J. A. Watt strain 1266/56A\&B; biotype A; United } \\
\text { Kingdom }\end{array}$ & 48,57 & 43.6 \\
\hline 52 & NCTC 10624 & P. haemolytica & $\begin{array}{l}\text { B. J. Shreeve strain } 7 \mathrm{H} 74 \mathrm{~L} ; \text { biotype T; serotype } 3 \text {; } \\
\text { lamb, United Kingdom }\end{array}$ & 57 & 42.6 \\
\hline 53 & SSI P737 & P. haemolytica & $\begin{array}{l}\text { W. Frederiksen; Frank, Ames strain 0-353-81; third } \\
\text { taxon; sheep, United States }\end{array}$ & 12 & 43.5 \\
\hline 54 & SSI P730 & P. haemolytica & $\begin{array}{l}\text { W. Frederiksen; Frank, Ames strain 0-305-80; } \\
\text { "intermedium strain"; sheep, United States }\end{array}$ & & 43.9 \\
\hline 55 & SSI P655 & P. haemolytica & W. Frederiksen; fourth taxon; pig, Denmark & & 41.4 \\
\hline 56 & NCTC $8141^{\mathrm{T}}$ & P. pneumotropica & $\begin{array}{l}\text { E. Jawetz strain M8.19.48; Frederiksen type } \\
\text { Jawetz; mouse, United States }\end{array}$ & 27,54 & 40.7 \\
\hline 57 & NCTC 8284 & P. pneumotropica & $\begin{array}{l}\text { M. T. Parker strain 54818; Frederiksen type Jawetz; } \\
\text { mouse, United Kingdom }\end{array}$ & & 42.8 \\
\hline 58 & HIM $640-5 / 6$ & $P$. pneumotropica & $\begin{array}{l}\text { O. Mraz strain P.u. } 2 ; \text { Frederiksen type Jawetz; } \\
\text { mouse, Czechoslovakia }\end{array}$ & 45 & 41.5 \\
\hline 59 & ATCC 12555 & $P$. pneumotropica & $\begin{array}{l}\text { R. G. Wyllie strain C57; Frederiksen type Heyl; } \\
\text { mouse, Australia }\end{array}$ & 16 & 40.3 \\
\hline 60 & HIM 769-1 & $P$. testudinis & $\begin{array}{l}\text { E. L. Biberstein strain UCD } 475-10 \text { A; tortoise, } \\
\text { United States }\end{array}$ & 61 & 47.0 \\
\hline 61 & ATCC $33688^{\mathrm{T}}$ & P. testudinis & $\begin{array}{l}\text { E. L. Biberstein strain UCD 90-23-79n; tortoise. } \\
\text { United States }\end{array}$ & 61 & 46.8 \\
\hline 62 & NCTC $10219^{\mathrm{T}}$ & P. ureae & S. D. Henriksen strain 3520/59: human, Norway & $22,23,54$ & 41.2 \\
\hline 63 & NCTC 10220 & P. ureae & S. D. Henriksen strain $218 / 60$; human, Norway & 22,23 & 43.7 \\
\hline 64 & SSI P602 & $\mathrm{SP}$ group & Altman strain 7098 ; human, Israel & 13 & 50.4 \\
\hline 65 & SSI P603 & $\mathrm{SP}$ group & W. Frederiksen strain SP1437; guinea pig, Denmark & 13 & 50.5 \\
\hline 66 & HIM 733-7 & SP group & $\begin{array}{l}\text { W. Mannheim strain Michel A/76; guinea pig, } \\
\text { Federal Republic of Germany }\end{array}$ & 40 & 52.5 \\
\hline 67 & ATCC 17911 & "P. piscicida" & $\begin{array}{l}\text { M. J. Pelczar strain } 315 \text {; epizootic of white perch, } \\
\text { United States }\end{array}$ & 60 & 39.5 \\
\hline 68 & NCTC 10699 & Pasteurella-like & $\begin{array}{l}\text { N. S. Mair strain } 5143 / 70 \text { : porcine fetus, United } \\
\text { Kingdom }\end{array}$ & & 43.4 \\
\hline 69 & SSI P335 & "P. multocida" & $\begin{array}{l}\text { W. Frederiksen strain SP5282-64; Frederiksen } \\
\text { biotype 1; guinea pig, Denmark }\end{array}$ & & 49.3 \\
\hline 70 & SSI P614 & "P. multocida" & $\begin{array}{l}\text { S. Namioka serotype 5:A; Frederiksen biotype 6; } \\
\text { chicken, Formosa }\end{array}$ & 47 & 40.9 \\
\hline 71 & NCTC 10547 & $\begin{array}{l}\text { Bovine lymphangitis } \\
\text { group }\end{array}$ & M. S. Jayaraman strain I; bovine, India & 28 & 49.7 \\
\hline 72 & NCTC 9509 & Y. pseudotuberculosis & E. Thal strain 105; mink, Denmark & 4 & 50.4 \\
\hline
\end{tabular}

" ATCC, American Type Culture Collection, Rockville, MD.; HIM, Culture Collection of the Zentrum für Hygiene und Medizinische Mikrobiologie, Marburg, Federal Republic of Germany: IPDH, Institute for Poultry Disease, Hannover, Federal Republic of Germany; NCTC. National Collection of Type Cultures, Central Public Health Laboratory, London, United Kingdom; SSI, W. Frederiksen, Statens Seruminstitut, Copenhagen, Denmark.

${ }^{b}$ The values are averages of six to nine assays. 

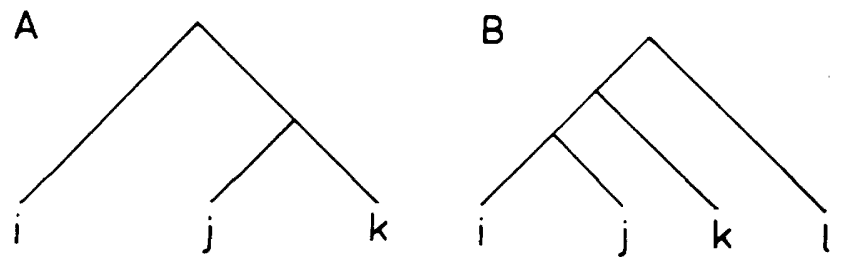

FIG. 1. Two possible dendrograms for OTUs $i$, $j$, and $k$ and OTUs $\mathbf{i}, \mathbf{j}, \mathbf{k}$, and $\mathbf{l}$. See text.

from each other by conventional methods (E. R. Hatt, R. Johnson, and R. L. Gherna, Abstr. Annu. Meet. Am. Soc. Microbiol. 1978, I54, p. 90).

These problems became even more complicated when phenotypic delineation of the genus Haemophilus Winslow et al. 1971 was considered, since V-factor dependency was no longer a criterion for separating genera (50).

In an effort to resolve some of these problems, we used deoxyribonucleic acid (DNA)-DNA hybridization to establish genetic relationships within the genus Pasteurella, the type genus of the family Pasteurellaceae (49; S. Pohl, Ph.D. thesis, Philipps-Universität, Marburg, Federal Republic of Germany, 1979) and separation of this genus from Actinobacillus and Haemophilus. DNA molecular weight and base composition were considered as additional taxonomic criteria.

In this study we included the recognized species of the three genera (54) and (especially for $P$. multocida), strains of the serogroups established by Carter (7) and the biotypes of Frederiksen (12) and a variety of isolates which represented phenotypic intermediates or possibly new species of the Pasteurellaceae.

\section{MATERIALS AND METHODS}

Microorganisms. The bacterial strains used in the DNA homology studies and their derivations are listed in Table 1.

Phenotypic characterization. The methods and media used to determine the phenotypic characteristics of the bacterial strains have been described previously $(38,40,41,45,50$, 62).

Cultivation techniques and preparation of DNA. Cells were grown aerobically at $35^{\circ} \mathrm{C}$ in Difco Proteose Peptone medium (68) supplemented with $0.4 \%(\mathrm{wt} / \mathrm{vol})$ filter-sterilized yeast extract (Oxoid Ltd., London, England) and $0.01 \mathrm{M}$ $\mathrm{NaHCO}_{3}$. The cells were harvested in the early stationary phase of growth, tested microscopically and culturally for purity, washed in $1 \times \mathrm{SSC}(0.15 \mathrm{M} \mathrm{NaCl}$ plus $0.015 \mathrm{M}$ trisodium citrate) and suspended in $1 \times$ SSC containing 0.01 $\mathrm{M}$ ethylenediaminetetraacetic acid (disodium salt). Highmolecular-weight DNA was isolated by combining the methods of Marmur (41) and Kirby et al. (30), as proposed by De Ley et al. (8).

Characterization of DNA. Thermal denaturation $\left(T_{m}\right)$ midpoints were used to determine the guanine-plus-cytosine $(\mathrm{G}+\mathrm{C})$ contents of the DNA preparations (42) (Table 1). The molecular weights of genome DNAs were determined from initial renaturation rates by using the method of Gillis et al. (15). Escherichia coli strain Luria B (= NCTC 10537) DNA was used as a reference. DNA preparations were further characterized by determining ultraviolet light spectra, protein contents (3), concentrations of 2-deoxyribose (6), and hyperchromicity.
DNA-DNA hybridization. Initial renaturation rates during homologous or heterologous reassociation were determined by the method of De Ley et al. (8), using a model 250 spectrophotometer (Gilford Instruments $\mathrm{GmbH}$, Düsseldorf, Federal Republic of Germany), a DNA concentration of 80 $\mu \mathrm{g} / \mathrm{ml}$, a salt milieu containing $2 \times \mathrm{SSC}$, and a reassociation temperature of $68^{\circ} \mathrm{C}$. The reassociation temperature was calculated as proposed by Gillis et al. (15). The reassociation method is supposed to be specific (i.e., to record only true base pairing); the DNA binding values (D values) obtained

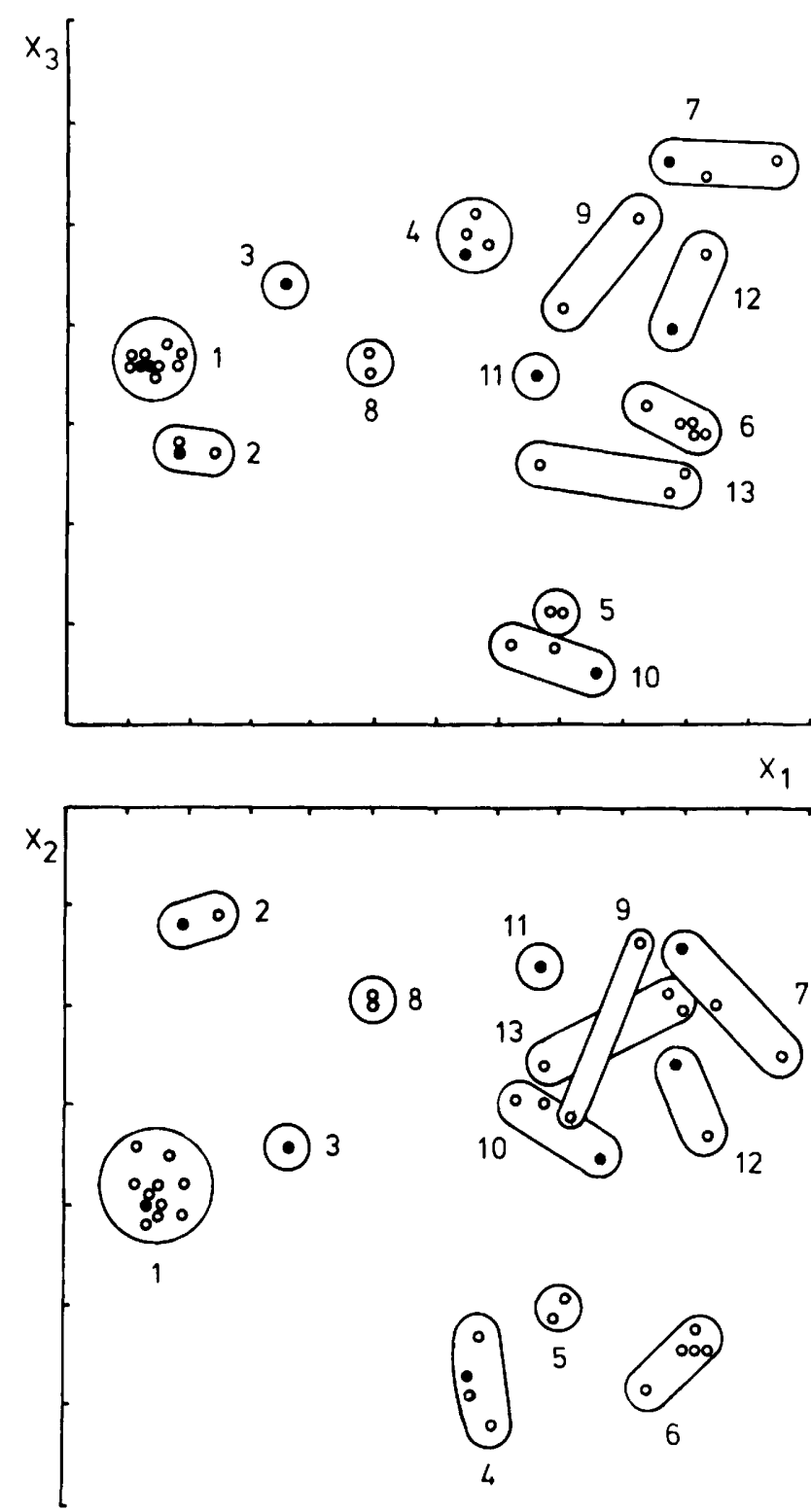

FIG. 2. Similarity map of the groups of the genus Pasteurella sensu stricto as revealed by principal component analysis. $x_{1}, x_{2}$, and $\mathrm{x}_{3}$ are coordinates of the three-dimensional Euclidean space. Symbols: position of two or more strains; $O$, position of one strain. The positions of taxa are indicated by numbers, as follows: 1 , $P$. multocida subsp. multocida $; 2, P$. multocida subsp. septica; $3, P$. multocida subsp. gallicida; $4, P$. dagmatis; $5, P$. gallinarum; $6, P$. canis; 7, P. stomatis; $8, P$. anatis; 9, Pasteurella species $\mathrm{B} ; 10$, Pasteurella species A; 11, $P$. langaa; $12, P$. avium; $13, P$. volantium. 
TABLE 2. D values obtained with species belonging to the genus Pasteurella

\begin{tabular}{|c|c|c|c|c|c|}
\hline \multirow{2}{*}{ Reference strain(s) ${ }^{a}$} & \multicolumn{2}{|c|}{ DNA binding } & \multirow{2}{*}{ Reference strain $(\mathrm{s})^{a}$} & \multicolumn{2}{|l|}{ DNA binding } \\
\hline & Strain $"$ & $\mathrm{D}$ value $(\%)$ & & Strain $^{a t}$ & D value $(\%)$ \\
\hline \multirow[t]{4}{*}{$\begin{array}{l}P . \text { multocida subsp. } \\
\text { multocida } 1\end{array}$} & $\begin{array}{l}\text { P. multocida subsp. } \\
\text { multocida } 2\end{array}$ & 100 & $\begin{array}{l}\text { P. multocida subsp. } \\
\text { multocida } 1\end{array}$ & $\begin{array}{l}P . \text { pneumotropica type } \\
\text { Jawetz } 56\end{array}$ & 28 \\
\hline & $\begin{array}{l}\text { P. multocida subsp. } \\
\text { multocida } 3\end{array}$ & 100 & & $\begin{array}{l}\text { P. multocida biotype } 1 \\
\text { strain } 69\end{array}$ & 27 \\
\hline & $\begin{array}{l}P . \text { multocida subsp. } \\
\text { multocida } 4\end{array}$ & 100 & & $\begin{array}{l}\text { P. pneumotropica type } \\
\text { Heyl } 59\end{array}$ & 27 \\
\hline & $\begin{array}{l}\text { P. multocida subsp. } \\
\text { multocida } 5\end{array}$ & 100 & $\begin{array}{l}\text { P. multocida subsp. } \\
\text { multocida } 9\end{array}$ & $\begin{array}{l}\text { P. pneumotropica type } \\
\text { Hey1 } 59\end{array}$ & 23 \\
\hline \multirow{2}{*}{$\begin{array}{l}\text { P. multocida subsp. } \\
\text { multocida } 8 \\
\text { P. multocida subsp. } \\
\text { multocida } 3\end{array}$} & $\begin{array}{l}\text { P. multocida subsp. } \\
\text { multocida } 5\end{array}$ & 95 & P. multocida subsp. & $\begin{array}{l}\text { "P. bettii" } 50 \\
P . \text { avium } 42\end{array}$ & $\begin{array}{l}26 \\
22\end{array}$ \\
\hline & $\begin{array}{l}P . \text { multocida subsp. } \\
\text { multocida } 7\end{array}$ & 100 & multocida 1 & P. langaa 40 & 20 \\
\hline \multirow[t]{2}{*}{$\begin{array}{l}P . \text { multocida subsp. } \\
\text { multocida } 1\end{array}$} & $\begin{array}{l}P . \text { multocida subsp. } \\
\text { multocida } 7\end{array}$ & 95 & & $\begin{array}{l}P \text {. canis } 28 \\
P \text {. stomatis } 33\end{array}$ & $\begin{array}{l}17 \\
17\end{array}$ \\
\hline & $\begin{array}{l}P . \text { multocida subsp. } \\
\text { multocida } 6\end{array}$ & 97 & & $\begin{array}{l}\text { P. stomatis } 34 \\
\text { Pasteurella-like SP group }\end{array}$ & $\begin{array}{l}16 \\
16\end{array}$ \\
\hline $\begin{array}{l}\text { P. multocida subsp. } \\
\text { multocida } 11\end{array}$ & $\begin{array}{l}\text { P. multocida subsp. } \\
\text { multocida } 6\end{array}$ & 97 & & $\begin{array}{l}\text { strain } 64 \\
P . \text { ureae } 62\end{array}$ & 15 \\
\hline $\begin{array}{l}\text { P. multocida subsp. } \\
\text { multocida } 5\end{array}$ & $\begin{array}{l}\text { P. multocida subsp. } \\
\text { multocida } 8\end{array}$ & 95 & $\begin{array}{l}P . \text { multocida subsp. } \\
\text { multocida } 9\end{array}$ & $P$. ureae 62 & 12 \\
\hline $\begin{array}{l}\text { P. multocida subsp. } \\
\text { multocida } 10\end{array}$ & $\begin{array}{l}\text { P. multocida subsp. } \\
\text { multocida } 8\end{array}$ & 94 & & $\begin{array}{l}P \text {. haemolytica biotype A } \\
\text { strain } 51\end{array}$ & 13 \\
\hline $\begin{array}{l}\text { P. multocida subsp. } \\
\text { multocida } 1\end{array}$ & $\begin{array}{l}P . \text { multocida subsp. } \\
\text { multocida } 8\end{array}$ & 92 & $\begin{array}{l}P . \text { multocida subsp. } \\
\text { multocida } 1\end{array}$ & P. testudinis 60 & 9 \\
\hline $\begin{array}{l}\text { P. multocida subsp. } \\
\text { multocida } 6\end{array}$ & $\begin{array}{l}\text { P. multocida subsp. } \\
\text { multocida } 11\end{array}$ & 97 & $\begin{array}{l}\text { P. multocida subsp. } \\
\text { multocida } 9\end{array}$ & P. volantium 45 & 6 \\
\hline $\begin{array}{l}\text { P. multocida subsp. } \\
\text { multocida } 1\end{array}$ & $\begin{array}{l}\text { P. multocida subsp. } \\
\text { multocida } 11\end{array}$ & 84 & & $\begin{array}{l}\text { Pasteurella-like bovine } \\
\text { lymphangitis group }\end{array}$ & 6 \\
\hline \multirow{3}{*}{$\begin{array}{l}\text { P. multocida subsp. } \\
\text { multocida } 8 \\
\text { P. multocida subsp. } \\
\text { multocida } 1\end{array}$} & $\begin{array}{l}\text { P. multocida subsp. } \\
\text { multocida } 10 \\
\text { multocida }\end{array}$ & 94 & P. multocida subsp. & $\begin{array}{l}\text { strain } 71 \\
\text { Pasteurella species B } \\
\text { strain } 38\end{array}$ & 4 \\
\hline & $\begin{array}{l}\text { P. multocida subsp. } \\
\text { multocida } 10\end{array}$ & 84 & & $\begin{array}{l}\text { stran } 38 \\
P . \text { stomatis } 35\end{array}$ & 3 \\
\hline & $\begin{array}{l}\text { P. multocida subsp. } \\
\text { multocida } 9\end{array}$ & 91 & & $\begin{array}{l}\text { Pasteurella-like Mair } \\
\text { strain } 68\end{array}$ & 1 \\
\hline $\begin{array}{l}\text { P. multocida subsp. } \\
\text { multocida } 11\end{array}$ & $\begin{array}{l}\text { P. multocida subsp. } \\
\text { septica } 15\end{array}$ & 82 & $\begin{array}{l}\text { P. multocida subsp. } \\
\text { multocida } 9\end{array}$ & $\begin{array}{l}\text { Pasteurella-like Mair } \\
\text { strain } 68\end{array}$ & 0 \\
\hline \multirow[t]{2}{*}{$\begin{array}{l}P . \text { multocida subsp. } \\
\text { multocida } 1\end{array}$} & $\begin{array}{l}\text { P. multocida subsp. } \\
\text { septica } 15\end{array}$ & 78 & $\begin{array}{l}P . \text { multocida subsp. } \\
\text { multocida } 1\end{array}$ & Y. pseudotuberculosis 72 & 1 \\
\hline & $\begin{array}{l}\text { P. multocida subsp. } \\
\text { septica } 12\end{array}$ & 80 & P. multocida subsp. & $\begin{array}{l}\text { P. aerogenes } 49 \\
\text { "P. piscicida" } 67\end{array}$ & $\begin{array}{l}0 \\
0\end{array}$ \\
\hline \multirow{2}{*}{$\begin{array}{l}\text { P. multocida subsp. } \\
\text { multocida } 8 \\
\text { P. multocida subsp. } \\
\text { multocida } 1\end{array}$} & $\begin{array}{l}\text { P. multocida subsp. } \\
\text { septica } 13\end{array}$ & 77 & $\begin{array}{l}\text { multocida } 9 \\
\text { P. multocida subsp. }\end{array}$ & $P$. multocida subsp. & 100 \\
\hline & $\begin{array}{l}\text { P. multocida subsp. } \\
\text { gallicida } 16 \\
\text { P. multocida subsp. }\end{array}$ & 73 & $\begin{array}{l}\text { septica } 12 \\
\text { P. multocida subsp. } \\
\text { septica } 15\end{array}$ & $\begin{array}{l}\text { septica } 15 \\
\text { P. multocida } \text { subsp. } \\
\text { septica } 13\end{array}$ & 94 \\
\hline \multirow{6}{*}{$\begin{array}{l}\text { P. multocida subsp. } \\
\text { multocida } 9 \\
P . \text { multocida } \text { subsp. } \\
\text { multocida } 5 \\
\text { P. multocida subsp. } \\
\text { multocida } 1\end{array}$} & $\begin{array}{l}\text { septica } 14 \\
\text { P. multocida subsp. }\end{array}$ & 65 & & $\begin{array}{l}\text { P. multocida } \text { subsp. } \\
\text { septica } 14\end{array}$ & 94 \\
\hline & $\begin{array}{l}\text { septica } 14 \\
\text { P. multocida subsp. }\end{array}$ & 55 & & & 89 \\
\hline & $\begin{array}{l}\text { septica } 14 \\
\text { P. dagmatis } 19\end{array}$ & 62 & & $\begin{array}{l}\text { P. multocida subsp. } \\
\text { multocida } 11\end{array}$ & 82 \\
\hline & P. dagmatis 20 & 59 & $\begin{array}{l}\text { P. multocida subsp. } \\
\text { septica } 12\end{array}$ & $\begin{array}{l}\text { P. multocida subsp. } \\
\text { multocida } 1\end{array}$ & 80 \\
\hline & $\begin{array}{l}\text { P. volantium } 45 \\
P . \text { volantium } 46\end{array}$ & $\begin{array}{l}59 \\
43\end{array}$ & $\begin{array}{l}\text { P. multocida subsp. } \\
\text { septica } 15\end{array}$ & $\begin{array}{l}\text { P. multocida subsp. } \\
\text { multocida } 1\end{array}$ & 78 \\
\hline & P. anatis 36 & 54 & $\begin{array}{l}\text { P. multocida subsp. } \\
\text { septica } 14\end{array}$ & P. multocida subsp. & 73 \\
\hline \multirow{2}{*}{$\begin{array}{l}P . \text { multocida subsp. } \\
\text { multocida } 9 \\
\text { P. multocida subsp. } \\
\text { multocida } 1\end{array}$} & P. gallinarum 25 & 40 & $\begin{array}{l}\text { septlca } 14 \\
\text { P. multocida subsp. } \\
\text { septica } 13\end{array}$ & $\begin{array}{l}\text { multociaa } 1 \\
\text { multocida subsp. } \\
\text { multocida } 8\end{array}$ & 77 \\
\hline & P. canis 27 & 33 & & $\begin{array}{l}\text { P. multocida subsp. } \\
\text { gallicida } 16\end{array}$ & 67 \\
\hline $\begin{array}{l}P . \text { multocida subsp. } \\
\text { multocida } 5\end{array}$ & P. canis 27 & 22 & $\begin{array}{l}\text { P. multocida subsp. } \\
\text { septica } 14\end{array}$ & $\begin{array}{l}\text { P. multocida subsp. } \\
\text { multocida } 9\end{array}$ & 65 \\
\hline \multirow{4}{*}{$\begin{array}{l}\text { P. multocida subsp. } \\
\text { multocida } 1 \\
\text { P. multocida subsp. } \\
\text { multocida } 3 \text { and } 11 \\
P . \text { multocida subsp. } \\
\text { multocida } 7 \\
\text { P. multocida subsp. } \\
\text { multocida } 9 \\
\end{array}$} & P. canis 27 & 21 & & $\begin{array}{l}\text { P. multocida subsp. } \\
\text { multocida } 5\end{array}$ & 55 \\
\hline & $P$ canis 27 & 18 & P. multocida subsp. & $P$. anatis 36 & 49 \\
\hline & T. cants 27 & 10 & P. multocida subsp. & P. multocida biotype 6 & 31 \\
\hline & $\begin{array}{l}\text { P. pneumotropica type } \\
\text { Jawetz } 57\end{array}$ & 32 & septica 15 & strain 70 & \\
\hline
\end{tabular}


TABLE 2-Continued

\begin{tabular}{|c|c|c|c|c|c|}
\hline \multirow{2}{*}{ Reference strain(s) ${ }^{a}$} & \multicolumn{2}{|l|}{ DNA binding } & \multirow{2}{*}{ Reference strain $(\mathrm{s})^{a}$} & \multicolumn{2}{|l|}{ DNA binding } \\
\hline & Strain $^{a}$ & D value $(\%)$ & & Strain $^{\prime \prime}$ & D value $(\%)$ \\
\hline \multirow{5}{*}{$\begin{array}{l}\text { P. multocida } \text { subsp. } \\
\text { septica } 13 \\
P . \text { multocida subsp. } \\
\text { septica } 15\end{array}$} & P. langaa 40 & $25^{b}$ & P. gallinarum 25 & P. avium 42 & $52^{b}$ \\
\hline & P. stomatis 33 & 25 & & $\begin{array}{l}\text { P. multocida subsp. } \\
\text { multocida } 9\end{array}$ & 42 \\
\hline & $P$. stomatis 35 & 13 & P. gallinarum 26 & $P$. haemolytica biotype $\mathrm{T}$ & 33 \\
\hline & P. dagmatis 24 & 10 & & strain 52 & \\
\hline & $P$. canis 27 & 1 & P. gallinarum 25 & P. multocida subsp. & 30 \\
\hline \multirow{8}{*}{$\begin{array}{l}P . \text { multocida subsp. } \\
\text { gallicida } 16\end{array}$} & P. multocida subsp. & 100 & & gallicida 16 & \\
\hline & $\begin{array}{l}\text { gallicida } 17 \\
\text { P. multocida subsp. }\end{array}$ & 91 & & $\begin{array}{l}\text { P. pneumotropica type } \\
\text { Jawetz strain } 57\end{array}$ & 28 \\
\hline & gallicida 18 & & & P. pneumotropica type & 25 \\
\hline & $\begin{array}{l}P . \text { multocida subsp. } \\
\text { multocida } 1\end{array}$ & 77 & & $\begin{array}{l}\text { Hey1 strain } 59 \\
P . \text { ureae } 62\end{array}$ & 21 \\
\hline & $\begin{array}{l}\text { P. multocida } \text { subsp. } \\
\text { septica } 12\end{array}$ & 67 & & $\begin{array}{l}P . \text { stomatis } 33 \\
P \text {. langaa } 40\end{array}$ & 20 \\
\hline & $\begin{array}{l}\text { Pasteurella } \text { species B } \\
\text { strain } 39\end{array}$ & 39 & P. gallinarum 26 & $\begin{array}{l}P \text {. haemolytica biotype A } \\
\text { strain } 51\end{array}$ & 20 \\
\hline & $\begin{array}{l}\text { Pasteurella species B } \\
\text { strain } 38\end{array}$ & 38 & P. gallinarum 25 & $\begin{array}{l}P . \text { aerogenes } 48 \\
P \text {. anatis } 36\end{array}$ & $\begin{array}{l}18 \\
13^{b}\end{array}$ \\
\hline & P. gallinarum 25 & 30 & & Pasteurella species B & 3 \\
\hline P. dagmatis 19 & P. dagmatis 21 & 100 & & strain 38 & \\
\hline P. dagmatis 22 & P. dagmatis 23 & 100 & P. canis 27 & P. canis 29 & 100 \\
\hline P. dagmatis 19 & $P$. dagmatis 23 & 91 & P. canis 30 & P. canis 28 & 100 \\
\hline P. dagmatis 24 & P. dagmatis 23 & 84 & P. canis 27 & $P$. canis 28 & 97 \\
\hline P. dagmatis 20 & $P$. dagmatis 24 & 100 & & P. canis 30 & 99 \\
\hline P. dagmatis 19 & $P$. dagmatis 24 & 85 & P. canis 30 & P. canis 31 & 92 \\
\hline P. dagmatis 23 & P. dagmatis 24 & 84 & P. canis 27 & P. canis 31 & 80 \\
\hline \multirow[t]{3}{*}{ P. dagmatis 19} & $P$. dagmatis 20 & 96 & P. canis 31 & $P$. avium 44 & 69 \\
\hline & P. dagmatis 22 & 92 & & Pasteurella species B & 62 \\
\hline & P. gallinarum 25 & 78 & & strain 39 & \\
\hline P. dagmatis 20 & P. gallinarum 26 & 72 & P. canis 27 & P. dagmatis 21 & 62 \\
\hline \multirow{2}{*}{$P_{j}$ dagmatis 19} & P. multocida subsp. & 62 & P. canis 29 & P. dagmatis 19 & 60 \\
\hline & multocida 1 & & P. canis 27 & P. dagmatis 19 & 43 \\
\hline \multirow[t]{2}{*}{ P. dagmatis 20} & P. multocida subsp. & 59 & & P. stomatis 35 & 54 \\
\hline & multocida 1 & & & P. stomatis 33 & 51 \\
\hline P. dagmatis 21 & P. canis 27 & 62 & & P. dagmatis 24 & 44 \\
\hline P. dagmatis 24 & P. canis 27 & 44 & & P. avium 43 & 41 \\
\hline \multirow[t]{2}{*}{ P. dagmatis 19} & P. canis 27 & 43 & P. canis 29 & P. multocida subsp. & 40 \\
\hline & P. canis 29 & 60 & & multocida 1 & \\
\hline P. dagmatis 24 & $P$. stomatis 33 & 59 & P. canis 27 & P. multocida subsp. & 22 \\
\hline P. dagmatis 19 & P. stomatis 33 & 40 & & multocida 1 & \\
\hline P. dagmatis 24 & P. stomatis 35 & 57 & P. canis 28 & P. multocida subsp. & 18 \\
\hline P. dagmatis 19 & $P$. volantium 45 & 43 & & multocida 1 & \\
\hline P. dagmatis 20 & P. volantium 45 & 25 & P. canis 27 & P. ureae 62 & 37 \\
\hline \multirow[t]{4}{*}{ P. dagmatis 19} & Pasteurella species B & 37 & P. canis 28 & P. ureae 62 & 31 \\
\hline & strain 38 & & & P. ureae 63 & 34 \\
\hline & $P$. avium 43 & 32 & P. canis 27 & $P$. multocida subsp. & 33 \\
\hline & P. langaa 40 & 30 & & multocida 5 & \\
\hline \multirow{4}{*}{ P. dagmatis 24} & P. anatis 36 & 29 & & P. stomatis 34 & 23 \\
\hline & P. multocida biotype 1 & 25 & & $P$. volantium 45 & 22 \\
\hline & strain 69 & & & P. multocida subsp. & 21 \\
\hline & $P$. ureae 62 & 22 & & multocida 11 & \\
\hline \multirow[t]{2}{*}{ P. dagmatis 19} & $P$. ureae 62 & 3 & & P. multocida subsp. & 21 \\
\hline & $P$. anatis 36 & 12 & & multocida 3 & \\
\hline P. dagmatis 24 & $\begin{array}{l}P . \text { multocida subsp. } \\
\text { septica } 15\end{array}$ & 10 & & $\begin{array}{l}\text { Pasteurella species B } \\
\text { strain } 38\end{array}$ & 19 \\
\hline \multirow{9}{*}{ P. dagmatis 19} & P. testudinis 60 & 6 & & P. multocida biotype 6 & 14 \\
\hline & P. pneumotropica type & 0 & & strain 70 & \\
\hline & Jawetz strain 56 & & & $P$. multocida subsp. & 12 \\
\hline & $P$. pneumotropica type & 0 & & multocida 9 & \\
\hline & Heyl strain 59 & & & $P$. pneumotropica type & 12 \\
\hline & $P$. aerogenes 48 & 0 & & Jawetz strain 56 & \\
\hline & $\begin{array}{l}\text { Pasteurella-like SP group } \\
\text { strain } 64\end{array}$ & 0 & & $\begin{array}{l}\text { P. multocida biotype } 1 \\
\text { strain } 69\end{array}$ & 10 \\
\hline & $\begin{array}{l}\text { Pasteurella-like SP group } \\
\quad \text { strain } 65\end{array}$ & 0 & & $\begin{array}{l}\text { P. multocida subsp. } \\
\text { septica } 15\end{array}$ & 1 \\
\hline & $Y$. pseudotuberculosis 72 & 0 & & "P. bettii" 50 & 0 \\
\hline$P$. gallinarum 25 & P. gallinarum 26 & 90 & P. stomatis 32 & P. stomatis 34 & 98 \\
\hline & P. dagmatis 19 & 78 & & $P$. stomatis 33 & 97 \\
\hline & P. volantium 45 & 72 & P. stomatis 33 & P. stomatis 35 & 91 \\
\hline P. gallinarum 26 & $P$. dagmatis 20 & 72 & P. stomatis 32 & $P$. avium 44 & 81 \\
\hline & P. avium 42 & 61 & P. stomatis 33 & P. avium 42 & 78 \\
\hline
\end{tabular}


TABLE 2-Continued

\begin{tabular}{|c|c|c|c|c|c|}
\hline \multirow{2}{*}{ Reference strain(s) ${ }^{a}$} & \multicolumn{2}{|l|}{ DNA binding } & \multirow{2}{*}{ Reference strain $(\mathrm{s})^{a}$} & \multicolumn{2}{|l|}{ DNA binding } \\
\hline & Strain $^{a}$ & $\mathrm{D}$ value $(\%)$ & & Strain $^{a}$ & $D$ value $(\%)$ \\
\hline \multirow[t]{2}{*}{ P. stomatis 32} & P. avium 43 & 74 & & P. gallinarum 25 & $20^{b}$ \\
\hline & $\begin{array}{l}\text { Pasteurella species B } \\
\text { strain } 38\end{array}$ & 63 & & $\begin{array}{l}P . \text { multocida subsp. } \\
\text { multocida } 1\end{array}$ & $20^{b}$ \\
\hline P. stomatis 33 & P. dagmatis 24 & 59 & & P. canis 27 & $14^{b}$ \\
\hline \multirow{2}{*}{ P. stomatis 35} & P. dagmatis 24 & 57 & & P. haemolytica biotype $\mathrm{T}$ & $10^{b}$ \\
\hline & P. canis 27 & 54 & & strain 52 & \\
\hline \multirow[t]{4}{*}{$P$. stomatis 33} & P. canis 27 & 51 & & P. haemolytica biotype A & $5^{b}$ \\
\hline & P. volantium 45 & 42 & & strain 51 & \\
\hline & P. dagmatis 19 & 40 & & P. avium 42 & $2^{b}$ \\
\hline & $P$. anatis 36 & 38 & & P. ureae 62 & 0 \\
\hline P. stomatis 35 & P. anatis 36 & 29 & P. avium 42 & P. avium 43 & 100 \\
\hline \multirow{2}{*}{$P$. stomatis 33} & P. multocida subsp. & 25 & & P. avium 44 & 88 \\
\hline & septica 15 & & P. avium 44 & P. stomatis 32 & 81 \\
\hline \multirow[t]{2}{*}{ P. stomatis 35} & P. multocida subsp. & 13 & P. avium 43 & P. stomatis 32 & 74 \\
\hline & septica 15 & & $P$. avium 42 & P. stomatis 33 & 78 \\
\hline \multirow[t]{2}{*}{ P. stomatis 34} & P. pneumotropica type & 22 & $P$. avium 44 & P. stomatis 31 & 69 \\
\hline & Jawetz strain 56 & & P. avium 42 & P. gallinarum 26 & 61 \\
\hline \multirow[t]{4}{*}{ P. stomatis 33} & $\begin{array}{l}\text { P. multocida biotype } 1 \\
\text { strain } 69\end{array}$ & 20 & & $\begin{array}{l}\text { P. volantium } 45 \\
\text { P. gallinarum } 25\end{array}$ & $\begin{array}{l}60^{b} \\
52^{b}\end{array}$ \\
\hline & P. gallinarum 25 & 20 & & P. volantium 47 & 44 \\
\hline & $P$. multocida subsp. & 17 & $P$. avium 43 & P. canis 27 & 41 \\
\hline & multocida 1 & & & P. dagmatis 19 & 32 \\
\hline P. stomatis 34 & $\begin{array}{l}\text { P. multocida subsp. } \\
\text { multocida } 1\end{array}$ & 16 & P. avium 42 & $\begin{array}{l}\text { Pasteurella species B } \\
\quad \text { strain } 38\end{array}$ & 29 \\
\hline P. stomatis 35 & $\begin{array}{l}P . \text { multocida subsp. } \\
\text { multocida } 1\end{array}$ & 3 & & $\begin{array}{l}\text { P. multocida subsp. } \\
\text { multocida } 1\end{array}$ & 22 \\
\hline P. stomatis 33 & $\begin{array}{l}\text { P. multocida biotype } 6 \\
\text { strain } 70\end{array}$ & 9 & & $\begin{array}{l}\text { P. anatis } 36 \\
\text { P. langaa } 40\end{array}$ & $\begin{array}{c}15 \\
2^{b}\end{array}$ \\
\hline \multirow[t]{3}{*}{ P. anatis 36} & $P$. anatis 37 & $94^{b}$ & P. volantium 45 & P. volantium 47 & 90 \\
\hline & P. multocida subsp & 54 & P. volantium 47 & $P$, volantium 46 & 88 \\
\hline & multocida 1 & & P. volantium 45 & P. volantium 46 & 81 \\
\hline P. anatis 37 & P. langaa 40 & 51 & & P. gallinarum 25 & 72 \\
\hline \multirow[t]{4}{*}{$P$. anatis 36} & P. langaa 40 & 36 & & P. gallinarum 26 & 70 \\
\hline & $P$. multocida subsp. & 49 & & P. avium 42 & 60 \\
\hline & septica 12 & & P. volantium 47 & P. avium 42 & 44 \\
\hline & P. stomatis 33 & 38 & P. volantium 45 & P. multocida subsp. & 59 \\
\hline P. anatis 37 & P. volantium 47 & 33 & & multocida 1 & \\
\hline \multirow{6}{*}{$P$. anatis 36} & P. stomatis 35 & 29 & P. volantium 47 & $P$. langaa 40 & 57 \\
\hline & P. dagmatis 24 & $29^{b}$ & P. volantium 45 & P. langaa 40 & 49 \\
\hline & P. volantium 45 & 18 & P. volantium 46 & Pasteurella species B & 52 \\
\hline & $P$. avium 42 & 15 & & strain 39 & \\
\hline & P. gallinarum 25 & $13^{b}$ & P. volantium 45 & P. dagmatis 19 & 43 \\
\hline & P. dagmatis 19 & 12 & P. volantium 46 & P. multocida subsp. & 43 \\
\hline \multirow{3}{*}{$\begin{array}{l}\text { Pasteurella species B } \\
\text { strain } 38\end{array}$} & Pasteurella species B & 87 & & multocida 9 & \\
\hline & strain 39 & & P. volantium 45 & $P$, stomatis 33 & 42 \\
\hline & $P$. stomatis 32 & 63 & P. volantium 47 & P. anatis 37 & 33 \\
\hline $\begin{array}{l}\text { Pasteurella species B } \\
\text { strain } 39\end{array}$ & P. canis 31 & 62 & P. volantium 45 & $\begin{array}{l}P \text {. pneumotropica type } \\
\text { Jawetz strain } 58\end{array}$ & 31 \\
\hline & $\begin{array}{l}\text { P. multocida subsp. } \\
\text { gallicida } 16\end{array}$ & 39 & P. volantium 46 & $\begin{array}{l}P \text {. pneumotropica type } \\
\text { Heyl strain } 59\end{array}$ & 29 \\
\hline $\begin{array}{l}\text { Pasteurella species B } \\
\text { strain } 38\end{array}$ & $\begin{array}{l}\text { P. multocida subsp. } \\
\text { gallicida } 16\end{array}$ & 38 & P. volantium 45 & $\begin{array}{l}P \text {. dagmatis } 20 \\
\text { "P. piscicida" } 67\end{array}$ & $\begin{array}{l}25 \\
24\end{array}$ \\
\hline & P. dagmatis 19 & 37 & P. volantium 46 & "P. piscicida" 67 & 20 \\
\hline & $P$. pneumotropica type & 30 & P. volantium 45 & P. canis 27 & 22 \\
\hline & Heyl strain 62 & & P. volantium 46 & Pasteurella-like Mair & 21 \\
\hline & P. avium 42 & 29 & & strain 68 & \\
\hline & P. canis 27 & 19 & P. volantium 45 & P. ureae 62 & 21 \\
\hline & P. multocida subsp. & 4 & & P. anatis 36 & 18 \\
\hline & multocida 1 & & P. volantium 46 & P. pneumotropica type & 17 \\
\hline & P. gallinarum 25 & 3 & & Jawetz strain 57 & \\
\hline & Y. pseudotuberculosis 72 & 0 & $P$. volantium 45 & P. pneumotropica type & 16 \\
\hline P. langaa 40 & P. langaa 41 & $100^{b}$ & & Jawetz strain 57 & \\
\hline & P. volantium 47 & 57 & & "P. bettii" 50 & 16 \\
\hline & P. anatis 37 & 51 & P. volantium 46 & "P. bettii" 50 & 0 \\
\hline & P. volantium 45 & $49^{b}$ & P. volantium 45 & Pasteurella-like Mair & 2 \\
\hline & $P$. anatis 36 & 36 & & strain 68 & \\
\hline & P. dagmatis 19 & $30^{h}$ & & Pasteurella-like bovine & 0 \\
\hline & $\begin{array}{l}\text { P. multocida subsp. } \\
\text { septica } 13\end{array}$ & $25^{b}$ & & $\begin{array}{l}\text { lymphangitis group } \\
\text { strain } 71\end{array}$ & \\
\hline
\end{tabular}

${ }^{a}$ Laboratory strain numbers (see Table 1).

${ }^{b}$ Unpublished data from K. Piechulla. 
by using this method correspond to the values determined with methods that detect reassociation of radioactively labeled DNA under stringent conditions.

Taxometric evaluation. The numerical methods used in bacterial taxonomy are based on a matrix of distance or similarity coefficients. The construction of the matrix is easy when each operational taxonomic unit (OTU) (59) is characterized by a vector of phenotypic characteristics or by a list of different base sequences, etc. In the case of DNA hybridization, however, each coefficient has to be measured directly. For $n$ OTUs, $n(n-1) / 2$ such coefficients would be needed, a quantity that is often too large to be realistic. Therefore, in most cases the distance or similarity matrix is incomplete. This is no handicap when the single-linkage method is used, but excludes other methods, such as the average-linkage method, the Ward method, or principal component analysis. Different methods have been proposed for completion of the matrix, with no one method being entirely satisfactory. Sneath (59) proposed that each pair of OTUs should be compared with a given number of reference strains. However, lack of information at the beginning of this study did not allow the definition of such strains. To carry out a principal component analysis, the similarity matrix was completed by interpolation. The following two methods were used simultaneously, depending on the $\mathrm{D}$ values: substitution and maximin interpolation.

(i) Substitution. $D_{i j} \geq 90 \%$ for OTUs $i$ and $j$; if only OTU $i$ is compared with OTU $k$ and not OTU $j$, then $D_{j k}=D_{i k}$.

(ii) Maximin interpolation. If $D_{i j}$ is undetermined, consider an OTU $k$ such that both $D_{i k}$ and $D_{j k}$ are known; then $D_{i j}=$ smaller value of $D_{i k}$ and $D_{j k}(\min )$. If there is more than one OTU (OTUs $k, 1, \ldots$ ), the greatest of $D_{i k}, D_{i l}, \ldots$ (max) has been taken.

The substitution method needs no further comments. The justification for the maximin interpolation method follows from Fig. 1, in which two possible dendrograms are shown for OTUs $\mathrm{i}, \mathrm{j}$, and $\mathrm{k}$ and OTUs $\mathrm{i}, \mathrm{j}, \mathrm{k}$, and $\mathrm{l}$. The smaller the stretch on the dendrogram from one OTU to another, the higher the similarity between the OTUs. In Fig. $1 \mathrm{~A}, \mathrm{D}_{\mathrm{ij}}=$ $D_{i k}$, the smaller of $D_{i k}$ and $D_{j k}$. In Fig. $1 B$, where $D_{i j}<D_{i k}$ and $D_{i l}$, the level of similarity is underestimated by this method. The possible error is lessened by using $D_{i k}$, the greater of $D_{i k}$ and $D_{i l}$. Thus, this method allows the conclusion that $D_{i j} \geq$ the maximal values of $D_{i k}, D_{i l}$, etc. Principal component analysis was carried out by using the Qtechnique. Each strain is represented as a point with three coordinates $\left(\mathrm{x}_{1}, \mathrm{x}_{2}\right.$, and $\left.\mathrm{x}_{3}\right)$ in an Euclidean space (Fig. 2).

\section{RESULTS AND DISCUSSION}

DNA reassociation data. The results of the DNA hybridization experiments are summarized in Tables 2 and 3 and Fig. 2.

The genus Pasteurella sensu stricto is restricted to organisms that cluster by single linkage above the 50\% DNA relatedness level (37). This proposal is in accordance with the principle of nomenclatural stability. If the genus was extended to lower DNA binding levels, the traditional genera and corresponding names (Actinobacillus, Haemophilus, and Pasteurella) could no longer be used $(36,37)$. On the other hand, well-defined species (Fig. 2) $(45,46)$ correspond to DNA relatedness clusters that are united at or above a DNA binding level of $85 \%$.

$P$. multocida. High relatedness values (90 to $100 \%$ DNA binding) were observed among the type strain of $P$. multocida (strain 1) and strains 2 through 11 (Table 1).
Additionally, strains belonging to the Carter serogroups (7) were included in this group, as described previously (62). The members of this group exhibited DNA binding levels of about $70 \%$ to another $P$. multocida group (namely, strains 12 through 15 , which were interrelated at a DNA binding level of more than $90 \%$ ). This second group could be differentiated from the former group by negative sorbitol fermentation.

A third taxonomic entity, which clustered at a DNA binding level of more than $90 \%$, consisted of $P$. multocida strains which fermented dulcitol and often L-arabinose (strains 16 through 18); this group was related to the two $P$. multocida groups described above at DNA binding levels of 80 and $70 \%$, respectively.

The DNA binding data would allow us to classify the three clusters described above as distinct species. However, this would be rather pointless from the clinical viewpoint. Therefore, we propose that $P$. multocida should be divided into three subspecies which may be useful for epidemiological purposes. The DNA homology group which includes the type strain, the representatives of the Carter serogroups, Frederiksen biotypes 2 to 5 , and strain 10, which was used in $16 \mathrm{~S}$ ribosomal ribonucleic acid studies of procaryotic phylogeny (69; L. B. Zablen, Ph.D. thesis, University of Illinois, Urbana, 1975), becomes $P$. multocida subsp. multocida. The cluster consisting of sorbitol-negative strains becomes $\boldsymbol{P}$. multocida subsp. septica, and the cluster consisting of dulcitol-positive strains becomes $P$. multocida subsp. gallicida. Thus, we preserve the main synonymy of $P$. multocida used in the past: the epithet septica is most frequently used in Anglo-Saxon countries, and the epithet gallicida is associated with the strains that ferment dulcitol and often arabinose. Rosenbusch and Merchant (53), as well as Dorsey (9) and Heddleston (20), described the fermentation of dulcitol and arabinose as characteristics of $P$. multocida strains isolated from avian sources. Variations in raffinose, lactose, maltose, trehalose, and $\mathrm{D}$-xylose fermentation were of no taxonomic consequence.

Another group in the genus Pasteurella is formed by the $P$. pneumotropica type Henriksen-Pasteurella "gas"-Pasteurella new species 1 group, represented by strains 19 through 24 . This group was shown to be related to $P$. gallinarum (strains 25 and 26) at a DNA binding level of $80 \%$ and to $P$. multocida at a level of $60 \%$. Based on correspondence between E. O. King and K. P. Carpenter from 1965 (K. P. Carpenter, personal communication), we propose the name Pasteurella dagmatis sp. nov. for this new species in the genus Pasteurella.

Pasteurella volantium Mutters et al. 1985 (46) (strains 45 through 47) was related to $P$. gallinarum at a DNA binding level of $70 \%$ and to $P$. dagmatis at a DNA binding level of $43 \%$.

Biotype 6 of $P$. multocida, represented by strains 27 through 30, exhibited high intragroup DNA relatedness (D value, more than $90 \%$ ); strain 31 , representing Bisgaard taxon $13\left(\mathrm{Orn}^{+}\right)(35 \mathrm{a})$ was also included in this taxon. This group exhibited D values of $69 \%$ to Pasteurella avium, $60 \%$ to $P$. dagmatis, and between 40 and $20 \%$ to $P$. multocida. Strains belonging to this group have also been designated "dog type" strains (12). We propose the name Pasteurella canis sp. nov. for this group because it is characteristically isolated from the throats of dogs or from human infections resulting from dog bites. For the needs of clinicians we propose division of this species into the following two biotypes: biotype 1 (indole positive, isolated from dogs and dog bite lesions) and biotype 2 (indole negative, isolated from calves). 
TABLE 3. D values obtained with species or groups not affiliated with the genus Pasteurella

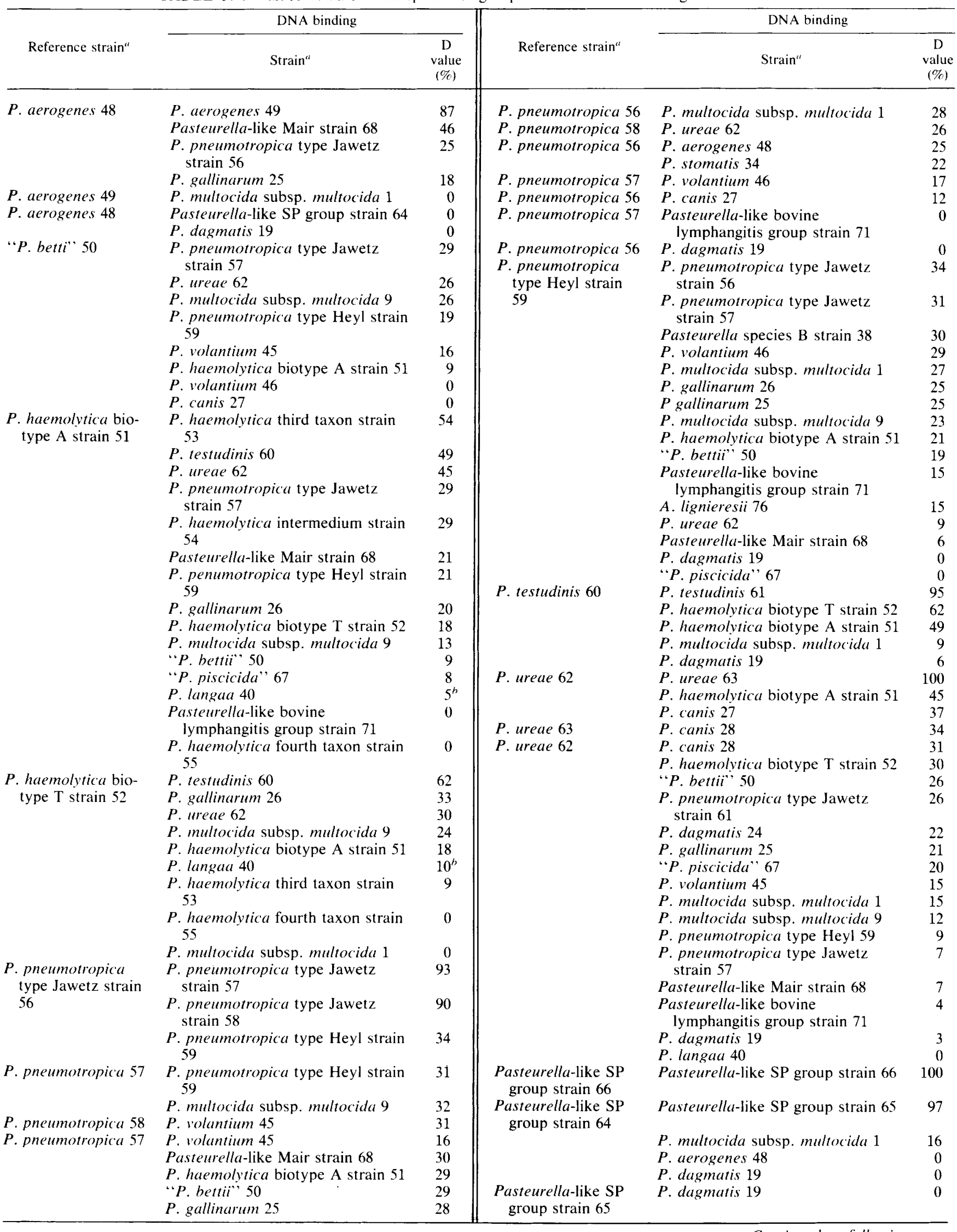


TABLE 3-Continued

\begin{tabular}{|c|c|c|}
\hline \multirow[b]{2}{*}{ Reference $\operatorname{strain}^{a}$} & \multicolumn{2}{|l|}{ DNA binding } \\
\hline & Strain $^{a}$ & $\begin{array}{c}\mathrm{D} \\
\text { value } \\
(\%)\end{array}$ \\
\hline \multirow[t]{6}{*}{ "P. piscicida" 67} & P. volantium 45 & 24 \\
\hline & P. ureae 62 & 20 \\
\hline & $P$. volantium 46 & 20 \\
\hline & P. haemolytica biotype A strain 51 & 8 \\
\hline & $\begin{array}{l}P . \text { pneumotropica type Heyl strain } \\
59\end{array}$ & 0 \\
\hline & P. multocida subsp. multocida 9 & 0 \\
\hline \multirow{11}{*}{$\begin{array}{l}\text { Pasteurella-like Mair } \\
\text { strain } 68\end{array}$} & P. aerogenes 48 & 46 \\
\hline & $\begin{array}{l}\text { P. pneumotropica type Jawetz } \\
\text { strain } 57\end{array}$ & 30 \\
\hline & P. haemolytica biotype A strain 51 & 21 \\
\hline & P. volantium 46 & 21 \\
\hline & P. multocida biotype 1 strain 69 & 12 \\
\hline & P. ureae 62 & 7 \\
\hline & $\begin{array}{l}P, \text { pneumotropica type Heyl strain } \\
59\end{array}$ & 6 \\
\hline & P. volantium 45 & 2 \\
\hline & P. multocida subsp. multocida 1 & 1 \\
\hline & Pasteurella-like SP group strain 64 & 0 \\
\hline & P. multocida subsp. multocida 9 & 0 \\
\hline \multirow{6}{*}{$\begin{array}{l}\text { Pasteurella-like bo- } \\
\text { vine lymphangitis } \\
\text { group strain } 71\end{array}$} & $\begin{array}{l}\text { P. pneumotropica type Heyl strain } \\
59\end{array}$ & 15 \\
\hline & P. multocida subsp. multocida 9 & 6 \\
\hline & P. testudinis 61 & 4 \\
\hline & P. volantium 45 & 0 \\
\hline & $\begin{array}{l}P \text {. pneumotropica type Jawetz } \\
\text { strain } 57\end{array}$ & 0 \\
\hline & P. haemolytica biotype A strain 51 & 0 \\
\hline
\end{tabular}

\footnotetext{
a Laboratory strain numbers (see Table 1).
}

${ }^{b}$ Unpublished data from K. Piechulla.

Another group showing high intragroup DNA homology (more than $90 \%$ ) is formed by strains 32 through 35 . This group is related to $P$. dagmatis at a DNA binding level of 40 to $60 \%$, to $P$. canis at a DNA binding level of 50 to $55 \%$, and to $P$. volantium at a DNA binding level of $42 \%$. With $P$. avium (Hinz and Kunjara) Mutters et al. 1985 (46), represented by strains 42 through 44 , D of about 74 to $80 \%$ were obtained.

Although this group shows fermentation reaction patterns similar to those of $P$. canis, it can be discriminated by its negative ornithine decarboxylase reaction. The DNA binding data indicate that this group is a species which is closely related to $P$. avium. We propose the name Pasteurella stomatis sp. nov. for this group; the normal habitat of these organisms is the throats, gums, and tonsils of mammals.

DNA-DNA hybridization experiments with dulcitolfermenting strains designated $P$. multocid $a$ biotype 6 (strains 38 and 39) indicated levels of DNA homology of $60 \%$ with $P$. stomatis and $P$. canis and about $40 \%$ with $P$. dagmatis and $P$. multocida subsp. gallicida. Despite the small number of strains investigated, these organisms should be tentatively regarded as a distinct species within the genus Pasteurella sensu stricto. For the present this species remains unnamed (provisionally called Pasteurella species B), since its clinical significance is unknown and a clear separation by computer analysis was not possible on the basis of the present data.

Bisgaard taxa 1 and 4 (1), represented by strains 36 and 37 and strains 40 and 41 , respectively, are interrelated at a DNA binding level of $51 \%$. Taxon 1 exhibits $54 \%$ DNA homology with $P$. multocida, and taxon 4 exhibits $57 \%$ homology with $P$. volantium. Thus, these two taxa, which were isolated from chickens and ducks, can be regarded as species of Pasteurella.

We propose the following names for these organisms: Pasteurella anatis sp. nov., for the facultatively pathogenic species represented by taxon 1, and Pasteurella langaa sp. nov., for the species consisting of taxon 4 .

A description of another new unnamed species of Pasteurella consisting of $\mathrm{L}$-arabinose-positive strains (provisionally called Pasteurella species A) has been presented previously (46).

Organisms exhibiting less than 50\% DNA relatedness to Pasteurella sensu stricto. Levels of DNA homology below $40 \%$ have been observed between species of the genus Pasteurella sensu stricto and representatives of organisms designated $P$. aerogenes, $P$. haemolytica, $P$. pneumotropica types Jawetz and Heyl, $P$. ureae, $P$. testudinis, and "Pasteurella piscicida." Between species of Pasteurella sensu stricto and members of the bovine lymphangitis group, the SP group, and group HB-5, binding values less than $30 \%$ or no measurable DNA binding was observed. DNA-DNA hybridization experiments with $P$. multocida biotype 1 (strain 69) and one biotype 6 strain (strain 70), which differed from other biotype 6 strains by its inability to ferment sucrose, yielded low or no measurable relationships to other Pasteurella strains.

A comparison of the species and groups described above with representative members of the Actinobacillus group yielded D values for $P$. ureae (strains 62 and 63 ) compared with Actinobacillus equuli NCTC $8529^{\mathrm{T}}$ ( $\mathrm{T}=$ type strain) and Actinobacillus lignieresii NCTC 4976 of 66 and 53\%, respectively. The level of DNA homology between $P$. ureae strain 62 and $P$. canis strain 27 was $40 \%$, and the level of homology between $P$. ureae and the type strain of $P$. multocida (strain 1) was $15 \%$.

$P$. haemolytica biotype A (strain 51) exhibited levels of DNA homology of 49 and $45 \%$ with $P$. testudinis (strain 60) and $P$. ureae, respectively. A level of DNA homology of $62 \%$ was observed between $P$. testudinis and $P$. haemolytica biotype T (strain 52). Thus, the two biotypes of $P$. haemolytica are interconnected via $P$. testudinis. The $P$. haemolytica group is more closely related to the genus Actinobacillus than to Pasteurella sensu stricto. It also includes strain 53, representing the third taxon of $P$. haemolytica (12). So far, the genetic data probably indicate the existence of a new genus-like structure within the family Pasteurellaceae, consisting of $P$. haemolytica biotypes A and $\mathrm{T}$, the third taxon, and $P$. testudinis. However, assignment of the "Actinobacillus salpingitidis" cluster to this group was not supported by DNA binding studies (K. Piechulla, personal communication).

Recently, $P$. pneumotropica types Jawetz and Heyl have been linked to the Actinobacillus group by DNA hybridization via two new species of Actinobacillus (R. Mutters and K. Piechulla, unpublished data).

DNA-DNA hybridization experiments with members of $P$. aerogenes, the SP group, group HB-5, the bovine lymphangitis group, " $P$. piscicida," and Actinobacillus or Haemophilus species did not solve the problems of the taxomic position of these groups. Only D values of less than $40 \%$ have been detected.

Compared with representatives of the genera Haemophilus and Actinobacillus (Actinobacillus capsulatus NCTC $11408^{\mathrm{T}}, A$. equuli NCTC $8529^{\mathrm{T}}$, A. lignieresii NCTC $4189^{\mathrm{T}}$ and NCTC 4976, Actinobacillus pleuropneumoniae CCM $5869^{\mathrm{T}}$, "A. salpingitidis" CCM 5974 ${ }^{\mathrm{T}}$ and CCM 5976, "Actinobacillus seminis" ATCC $15768^{\mathrm{T}}$, Actinobacillus suis 
CCM 5586 ${ }^{\mathrm{T}}$, Haemophilus aegyptius ATCC $11116^{\mathrm{T}}$, Haemophilus aphrophilus NCTC 5886, Haemophilus haemoglobinophilus $\mathrm{NCTC} 1659^{\mathrm{T}}$, Haemophilus influenzae NCTC $8143^{\mathrm{T}}$ and NCTC 4560, Haemophilus paragallinarum ATCC $29545^{\mathrm{T}}$, Haemophilus parahaemolyticus NCTC $8479^{\mathrm{T}}$, and Haemophilus parasuis NCTC $4557^{\mathrm{T}}$ ) the species of the genus Pasteurella sensu stricto showed levels of DNA homology of $40 \%$ or less. No measurable DNA binding has been observed among the type species of the three genera (P. multocida NCTC $10322^{\mathrm{T}}$, A. lignieresii $\mathrm{NCTC} 4189^{\mathrm{T}}$, and $H$. influenzae NCTC $8143^{\mathrm{T}}$ ).

A strain of Yersinia pseudotuberculosis type III (strain 72) was included in this study because it had been found to give a value of $28 \%$ with $P$. multocida under permissive conditions when the hydroxyapatite method was used (4). With the renaturation method, no measurable DNA binding between this Yersinia strain and representative strains of Pasteurella sensu stricto was detected. Thus, there is no evidence so far for a close connection between the families Pasteurellaceae and Enterobacteriaceae, as has also been observed by Ursing (67).

Phenotypic features. On the basis of the molecular data presented above, the genus Pasteurella sensu stricto can be differentiated from related genera by the features described below. The cells are coccobacillary to rod shaped and 0.3 to $0.4 \mu \mathrm{m}$ in diameter and appear singly, in pairs, or (less frequently) in short chains. They are not flagellated; endospores are not formed. The gram reaction is negative. The organisms are aerobic to microaerophilic or facultatively anaerobic and contain demethylmenaquinones (always) and ubiquinones (usually) but no menaquinones; glucose catabolism is fermentative. Nitrate is reduced. No growth occurs in Simmons citrate medium. All species are nonhemolytic; catalase is produced by most strains. The DNA base composition ranges from 37.7 to $45.9 \mathrm{~mol} \% \mathrm{G}+\mathrm{C}$; genome molecular weights are between $1.4 \times 10^{9}$ and $1.9 \times$ $10^{9}$.

Positive results are always observed for tetramethyl-pphenylenediamine oxidase and alkaline phosphatase; acid is produced within 24 to $48 \mathrm{~h}$ from D-glucose, D-galactose, D-fructose, D-mannose, and sucrose.

Negative results are always observed for arginine dihydrolase and lysine decarboxylase; acid is not produced from L-sorbose, L-rhamnose, $m$-inositol, adonitol, or salicin. Starch and esculin are not hydrolyzed.

The DNA binding clusters within the genus, which resemble the species and subspecies of Pasteurella, can be differentiated by the characteristics shown in Table 4 .

Reclassification of the genus Pasteurella Trevisan 1887. Considering the genetic relationships among the organisms investigated, we propose reclassification of the genus Pasteurella Trevisan 1887 and its species as shown below.

Genus Pasteurella Trevisan 1887. Pasteurella (Pas.teu.rel'la.M.L.dim.fem.n. Pasteurella named after Louis Pasteur) cells are nonmotile, small, and coccoid to rod shaped, and, depending on the growth stage, they occur singly, in pairs, or short chains. Gram reaction is negative. Not acid fast. Endospores are not formed.

Surface colonies on chocolate agar are round, grayish or yellowish, and nearly $2 \mathrm{~mm}$ in diameter after $48 \mathrm{~h}$. Colonies of some species have a rough character and may reach $1 \mathrm{~mm}$ in diameter. No hemolysis on sheep blood agar.

Mesophilic; chemoorganotrophic with both oxidative and fermentative types of metabolism. Ubiquinones and demethylmenaquinones are produced; molecular oxygen is used as a terminal electron acceptor but can be replaced by nitrate and fumarate. Oxidase positive, alkaline phosphatase positive, almost always catalase positive. Most species are $\mathrm{V}$-factor ( $\beta$-nicotinamide adenine dinucleotide) and $\mathrm{X}$-factor (protohemin or protoporphyrin IX) independent, although V-factor-requiring species and strains do occur.

Members of the genus are always promptly positive for fermentation of D-glucose, D-galactose, D-fructose, Dmannose, and sucrose; acid is not produced from L-sorbose, L-rhamnose, $m$-inositol, or adonitol. No hydrolysis of starch, salicin, or esculin occurs. Arginine dihydrolase, lysine decarboxylase, and liquefaction of gelatin are negative, except for a delayed gelatinase reaction that is observed after more than 14 days of incubation with strains of $P$. dagmatis (13). Fermentation of maltose is mostly combined with fermentation of trehalose. Members of the genus are parasitic in vertebrates, particularly mammals and birds.

The genome DNAs of members of the genus Pasteurella contain 37.7 to $45.9 \mathrm{~mol} \% \mathrm{G}+\mathrm{C}\left(T_{m}\right.$ method). The genome molecular weights range from $1.4 \times 10^{9}$ to $1.9 \times 10^{9}$.

Type species: Pasteurella multocida (Lehmann and Neumann, 1899) Rosenbusch and Merchant 1939.

The proposed species in the genus Pasteurella are described below.

Pasteurella multocida (Lehmann and Neumann 1899) Rosenbusch and Merchant 1939. Pasteurella multocida (mul.to.ci'da. L.adj. multus many; L.adj.suf. cidus kill; M.L.fem.adj. multocida many killing, referring to pathogenicity for many species of animals) is divided into the following three subspecies: $P$. multocida subsp. multocida, $P$. multocida subsp. septica (sep'ti.ca. M.L.fem.adj. septica poisoning, infecting), and $\boldsymbol{P}$. multocida subsp. gallicida (gal.li.ci'da. L.fem.n.gallina hen; L.adj.suf. cida kill; M.L.adj. gallicida hen killing, referring to pathogenicity for poultry).

In addition to the features consistent with all members of the genus, the biochemical reactions described below are common to the three subspecies. Acid is produced from mannitol. Positive ornithine decarboxylase reaction. Acid is produced from trehalose; production of acid from D-xylose differs in different strains. Exceptionally, acid is produced from raffinose, D-lactose, and (exceptionally) maltose. No $\mathrm{V}$-factor requirement. The $\mathrm{G}+\mathrm{C}$ contents of all subspecies range from 40.8 to $43.9 \mathrm{~mol} \%$ ( $T_{m}$ method). The molecular weights of genome DNAs range from $1.5 \times 10^{9}$ to $1.9 \times 10^{9}$. The main characteristics used for differentiation of the three subspecies are fermentation of sorbitol and dulcitol. $P$. multocida subsp. multocida strains ferment sorbitol but do not ferment dulcitol; $\boldsymbol{P}$. multocida subsp. septica strains exhibit negative reactions for both sorbitol and dulcitol fermentation, whereas the $P$. multocida subsp. gallicida strains ferment both sorbitol and dulcitol. Other reactions are shown in Table 4.

Strains of the species are isolated from most mammals, including humans and birds.

Strain NCTC 10322 is the type strain of $P$. multocida subsp. multocida. Strain CIP A125 is the type strain of $P$. multocida subsp. septica, and strain NCTC 10204 is type strain of $P$. multocida subsp. gallicida.

Pasteurella dagmatis sp. nov. Pasteurella dagmatis (dag.ma'tis. Gr.fem.gen.n. dagmatis from a bite) consists of strains that do not require V-factors. They produce small amounts of gas from D-glucose; positive reactions are obtained for urease, indole, acid production from maltose and, with few exceptions, acid production from trehalose. No acid is produced from D-xylose, $\mathrm{L}$-arabinose, raffinose, mannitol, sorbitol, or dulcitol. Ornithine is not decarboxylated. 
TABLE 4. Differential characteristics of the species belonging to Pasteurella sensu stricto

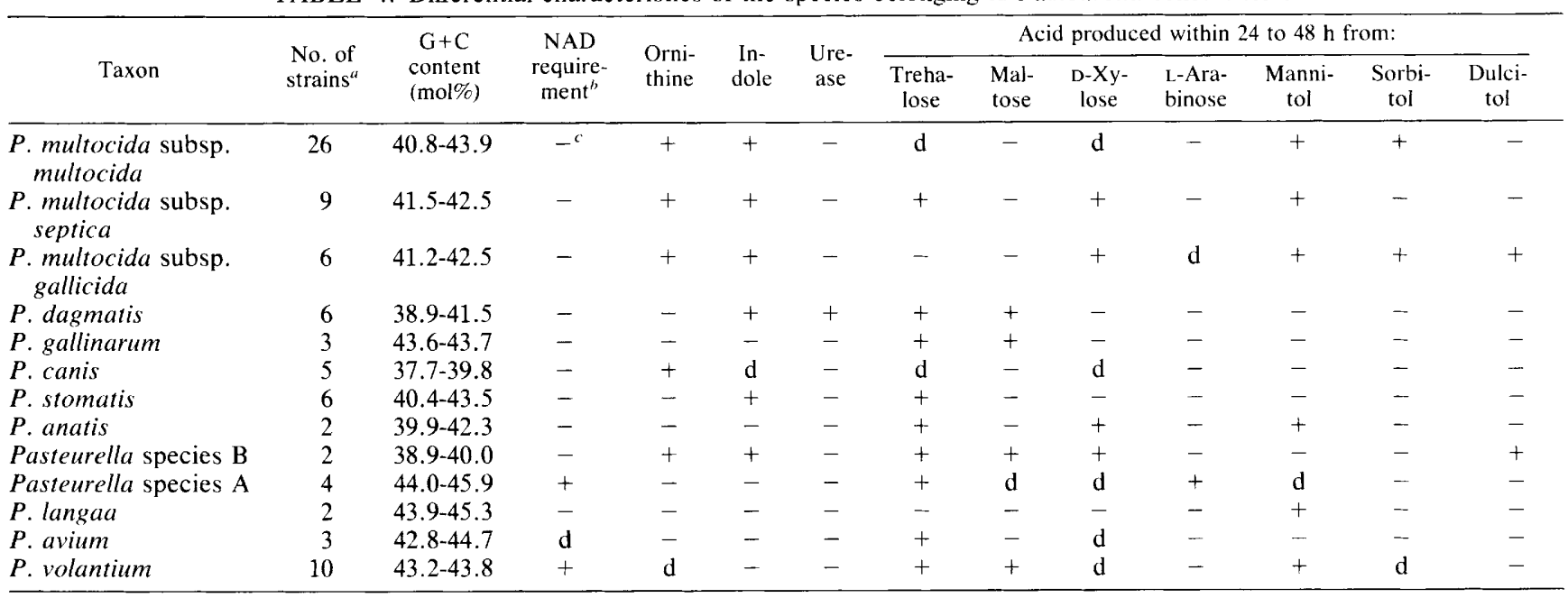

"Phenotypically identical strains that were not considered for DNA relatedness were included.

${ }^{b}$ NAD. Nicotinamide adenine dinucleotide.

$c+, \geq 90 \%$ of the strains are positive;,$- \geq 90 \%$ of the strains are negative; $d$, different results observed.

Gelatin may be liquefied after more than 14 days of incubation.

$P$. dagmatis strains have been isolated from dogs and cats, as well as from human local and systemic infections resulting from animal bites.

The $\mathrm{G}+\mathrm{C}$ content of the DNA ranges from 38.9 to 41.5 mol\% ( $T_{m}$ method); genome molecular weights range from $1.5 \times 10^{9}$ to $1.7 \times 10^{9}$.

The type strain of $P$. dagmatis is strain NCTC 11617 .

Pasteurella canis sp. nov. Pasteurella canis (ca'nis. L.gen.n. canis of a dog) strains are V-factor independent. The ornithine decarboxylation test is positive. Acid is not produced from L-arabinose, raffinose, D-lactose, maltose, mannitol, sorbitol, or dulcitol. The urease test is negative; variable reactions are obtained for acid production from trehalose and D-xylose. Biotype 1 strains exhibit positive reactions for indole; biotype 2 strains exhibit negative indole formation reactions.

$P$. canis biotype 1 is found in the oral cavities of dogs and is often isolated from injuries in humans resulting from dog bites. Biotype 2 strains have been isolated from calves. The $\mathrm{G}+\mathrm{C}$ content of the DNA ( $T_{m}$ method) is 37.7 to $39.8 \mathrm{~mol} \%$. The genome molecular weights range from $1.4 \times 10^{9}$ to $1.6 \times$ $10^{9}$.

The type strain of $P$. canis is strain NCTC 11621; the reference strain for biotype 2 is strain HIM 843-5.

Pasteurella stomatis sp. nov. Pasteurella stomatis (sto.ma'tis. Gr.gen.n. stomatis of the throat) strains fulfill the general criteria of the genus Pasteurella sensu stricto; in addition, they are V-factor independent, do not decarboxylate ornithine, and give negative urease tests. No acid is produced from L-arabinose, D-xylose, raffinose. D-lactose, maltose, trehalose, mannitol, sorbitol, or dulcitol. Indole and acid are produced from trehalose.

The strains of $P$. stomatis occur in the respiratory tracts of cats and dogs.

The $\mathrm{G}+\mathrm{C}$ content of the DNA ranges from 40.4 to 43.5 mol\% ( $T_{m}$ method). The genome molecular weights range from $1.5 \times 10^{9}$ to $1.6 \times 10^{9}$.

The type strain is strain NCTC 11623.

Pasteurella anatis sp. nov. Pasteurella anatis (a.na'tis.
L.fem.gen.n. anatis of a duck) strains have no V-factor requirement. They do not decarboxylate ornithine, do not produce indole, and do not hydrolyze urea. No acid is produced from L-arabinose, maltose, D-lactose, raffinose, sorbitol, or dulcitol; acid is produced from trehalose, Dxylose, and mannitol.

Members of this species have been isolated from the intestinal tracts of ducks.

The $\mathrm{G}+\mathrm{C}$ content is 39.9 to $42.3 \mathrm{~mol} \%$ ( $T_{m}$ method). The genome molecular weights range from $1.8 \times 10^{9}$ to $1.9 \times 10^{9}$.

The type strain of $P$. anatis is strain NCTC 11413 .

Pasteurella langaa sp. nov. Pasteurella langaa (lan'gaa. L.fem.n. langaa referring to Langaa, Denmark) has no $\mathrm{V}$-factor requirement and contains strains which were included in taxon 4 of Bisgaard (1). The members of this species produce acid from mannitol and lactose; tests for ornithine, indole, urease, acid from L-arabinose, D-xylose, raffinose, trehalose, maltose, and sorbitol are negative.

Strains of this species are found in the respiratory tracts of apparently healthy chickens.

The $\mathrm{G}+\mathrm{C}$ content of the DNA ranges from 43.9 to 45.3 mol\%, and the genome molecular weights range from $1.7 \times$ $10^{9}$ to $1.9 \times 10^{9}$

The type strain is strain NCTC 11411.

Unnamed new Pasteurella species B should be described when more strains have been studied. We suggest strain SSI $\mathrm{P} 683$ as the reference strain for this group.

$P$. gallinarum corresponds to the original description of Hall et al. (16). However, our data suggest that tests for mannitol, sorbitol, and $m$-inositol fermentation are negative, rather than irregular.

$P$. avium, $P$. volantium, and an additional new V-factordependent species (provisionally called Pasteurella species A) have been described previously (46).

\section{ACKNOWLEDGMENTS}

We are indebted to all who supplied valuable cultures and to Karl Piechulla for helpful discussions. The excellent technical assistance of Anneliese Hoops and Rita Diehl is acknowledged.

This work was supported in part by a grant from the Deutsche Forschungsgemeinschaft to W.M. 


\section{LITERATURE CITED}

1. Bisgaard, M. 1982. Isolation and characterization of some previously unreported taxa from poultry with phenotypical characters related to Actinobacillus and Pasteurella species. Acta Pathol. Microbiol. Immunol. Sect. B 90:59-67.

2. Bisgaard, M., R. Mutters, and W. Mannheim. 1983. Characterization of some previously unreported taxa isolated from guinea pigs (Cavia porcellus) and provisionally classed with the "HPAgroup." Les editions INSERM 114:227-244.

3. Bradford, M. M. 1976. A rapid and sensitive method for the quantitation of microgram quantities of protein utilizing the principle of protein-dye binding. Anal. Biochem. 72:248-254.

4. Brenner, D. J., A. G. Steigerwalt, D. P. Falcáo, R. E. Weaver, and R. Fanning. 1976. Characterization of Yersinia enterocolitica and Yersinia pseudotuberculosis by deoxyribonucleic acid hybridization and by biochemical reactions. Int. J. Syst. Bacteriol. 26:180-194.

5. Burrill, T. J. 1883. New species of Micrococcus (bacteria). Am. Nat. 17:319-320.

6. Burton, K. 1956. A study of the conditions and mechanisms of the diphenylamine reaction for the colorimetric estimation of deoxyribonucleic acid. Biochem. J. 62:315-322.

7. Carter, G. R. 1955. Studies on Pasteurella multocida. I. A hemagglutination test for the identification of serological types. Am. J. Vet. Res. 16:481-484.

8. De Ley, J., H. Cattoir, and A. Reynaerts. 1970. The quantitative measurement of DNA hybridization from renaturation rates. Eur. J. Biochem. 12:133-142.

9. Dorsey, T. A. 1963. Studies on fowl cholera. I. A biochemic study of avian Pasteurella multocida strains. Avian Dis. 7:386-392.

10. Enderlein, G. 1917. Ein neues Bakteriensystem auf vergleichend morphologischer Grundlage (Bakteriologische Studien IV). Berl. Ges. Naturfreund. Berlin, p. 309-319.

11. Ferry, N. S., and H. P. Hoskins. 1920. Bacteriology and control of contagious nasal catarrh (snuffles of rabbits). J. Lab. Clin. Med. 5:311-318.

12. Frederiksen, W. 1973. Pasteurella taxonomy and nomenclature. Contrib. Microbiol. 2:170-176.

13. Frederiksen, W. 1981. Gas producing species within Pasteurella, and Actinobacillus, p. 185-196. In M. Kilian, W. Frederiksen, and E. L. Biberstein (ed.), Haemophilus, Pasteurella, and Actinobacillus. Academic Press, Inc., London.

14. Gamaléia, N. 1888. Zur Aetiologie der Hühnercholera. Zentralbl. Bakteriol. Parasitenkd. Infektionskr. Hyg. Abt. 1 Orig. 4:161-168.

15. Gillis, M., J. De Ley, and M. De Cleene. 1970. The determination of molecular weight of bacterial genome DNA from renaturation rates. Eur. J. Biochem. 12:143-153.

16. Gray, D. F., and A. L. Campbell. 1953. The use of chloramphenicol and foster mothers in the control of natural pasteurellosis in experimental mice. Aust. J. Exp. Biol. Med. Sci. 31:161-165.

17. Gump, D. W., and R. A. Holden. 1972. Endocarditis caused by a new species of Pasteurella. Ann. Intern. Med. 76:275-278.

18. Haagsma, J. 1964. Een studie over urease bezittende Pasteurella multocida-stammen bij de kat in Nederland. Tijdschr. Diergeneeskd. 89:1005-1013.

19. Hall, W. J., K. L. Heddleston, D. H. Legenhausen, and R. W. Hughes. 1955. Studies on pasteurellosis. I. A new species of Pasteurella encountered in chronic fowl cholera. Am. J. Vet. Res. 16:598-604.

20. Heddleston, K. L. 1976. Physiologic characteristics of 1,268 cultures of Pasteurella multocida. Am. J. Vet. Res. 37:745-747.

21. Henriksen, S. D., and L. O. Frøholm. 1975. A fimbriated strain of Pasteurella multocida with spreading and corroding colonies. Acta Pathol. Microbiol. Scand. Sect. B 83:129-132

22. Henriksen, S. D., and K. Jyssum. 1960. Brief reports. A new variety of Pasteurella haemolytica from the human respiratory tract. Acta Pathol. Microbiol. Scand. 50:443.

23. Henriksen, S. D., and K. Jyssum. 1961. A study of some Pasteurella strains from the human respiratory tract. Acta
Pathol. Microbiol. Scand. 51:354-368.

24. Hinz, K.-H., and C. Kunjara. 1977. Haemophilus avium, a new species from chickens. Int. J. Syst. Bacteriol. 27:324-329.

25. Hoschatt, H., and W. Mannheim. 1979. On the phenotypical characteristics of human Pasteurella, and Pasteurella-like isolates. Zentralbl. Bakteriol. Hyg. Abt. 1 Orig. Reihe A 243:499-510.

26. Hussaini, S. N. 1975. Nomenclature and taxonomy of Pasteurella multocida. Vet. Bull. 45:403-409.

27. Jawetz, E. 1950. A pneumotropic pasteurella of laboratory animals. 1. Bacteriological and serological characteristics of the organism. J. Infect. Dis. 86:172-183.

28. Jayaraman, M. S., and V. Sethumadhavan. 1974. The B.L. organism-the causal agent of bovine lymphangitis in Tamil Nadu. Indian Vet. J. 51:347-355.

29. Jones, D. M. 1962. A pasteurella-like organism from the human respiratory tract. J. Pathol. Bacteriol. 83:143-151.

30. Kirby, K. S., E. Fox-Carter, and M. Guest. 1967. Isolation of deoxyribonucleic acid and ribosomal ribonucleic acid from bacteria. Biochem. J. 104:258-262.

31. Kitt, T. 1885. Uber eine experimentelle, der Rinderseuche ähnliche Infektionskrankheit. Sitzungsber. Ges. Morphol. Physiol. Muenchen, p. 140-168.

32. Kitt, T. 1893. Septicaemia haemorrhagica, p. 302-308. In T. Kitt (ed.), Bacterienkunde und pathologische Mikroskopie für Thierärzte und Studierende der Thiermedizin, 2nd ed. Moritz Perles, Vienna.

33. Kitt, T. 1903. Septicaemia haemorrhagica s. pluriformis, p. 559-565. In W. Kolle and A. von Wassermann (ed.), Handbuch der pathogenen Mikroorganismen, 1st ed., vol. 2. Gustav Fischer, Jena.

34. Lehmann, K. B., and R. Neumann. 1899. Lehmann's Medizin. Handatlanten, X. Atlas mit Grundriss der Bakteriologie und Lehrbuch der Speziellen Bakteriologischen Diagnostik, 3rd ed. J. F. Lehmann, Munich.

35. Lignières, M. J. 1901. Contribution a L'étude et la classification des septicémies hémorrhagiques, les "pasteurelloses." Ann. Inst. Pasteur Paris 10:734-736.

35a.Madsen, E. B., M. Bisgaard, R. Mutters, and K. B. Pedersen. 1985. Characterization of Pasteurella spp. isolated from lungs of calves with pneumonia. Can. J. Comp. 49:63-67.

36. Mannheim, W. 1981. Taxonomic implications of DNA relatedness and quinone patterns in Actinobacillus, Haemophilus, and Pasteurella, p. 265-280. In M. Kilian, W. Frederiksen, and E. L. Biberstein (ed.), Haemophilus, Pasteurella, and Actinobacillus. Academic Press, Inc., London.

37. Mannheim, W. 1983. Taxonomy of the family Pasteurellaceae Pohl 1981 as revealed by DNA/DNA hybridization. INSERM 114:211-226.

38. Mannheim, W., S. Pohl, and R. Holländer. 1980. On the taxonomy of Actinobacillus, Haemophilus, and Pasteurella: DNA base composition, respiratory quinones, and biochemical reactions of representative collection cultures. Zentralbl. Bakteriol. Hyg. Abt. 1 Orig. Reihe A 246:512-538.

39. Mannheim, W., S. Pohl, and W. Stenzel. 1978. Unclassified Pasteurella-like organisms isolated from guinea pigs. Zentralbl. Bakteriol. Hyg. Abt. 1 Orig. Reihe A 241:329-336.

40. Mannheim, W., W. Stieler, G. Wolf, and R. Zabel. 1978. Taxonomic significance of respiratory quinones and fumarate respiration in Actinobacillus and Pasteurella. Int. J. Syst. Bacteriol. 28:7-13.

41. Marmur, J. 1961. A procedure for the isolation of deoxyribonucleic acid from micro-organisms. J. Mol. Biol. 3:208-218.

42. Marmur, J., and P. Doty. 1962. Determination of the base composition of deoxyribonucleic acid from its thermal denaturation temperature. J. Mol. Biol. 5:109-118.

43. McAllister, H. A., and G. R. Carter. 1974. An aerogenic Pasteurella-like organism recovered from swine. Am. J. Vet. Res. 35:917-922.

44. Mráz, O. 1969. Vergleichende Studie der Arten Actinobacillus lignieresii und Pasteurella haemolytica. III. Actinobacillus haemolyticus (Newsom and Cross, 1932) comb. nov. Zentralbl. Bakteriol. Parasitenkd. Infektionskr. Hyg. Abt. 1 Orig. 
209:349-364.

45. Mutters, R., W. Frederiksen, and W. Mannheim. 1984. Lack of evidence for the occurrence of Pasteurella ureae in rodents. Vet. Microbiol. 9:83-93.

46. Mutters, R., K. Piechulla, K.-H. Hinz, and W. Mannheim. 1985. Pasteurella avium (Hinz and Kunjara) comb. nov. and Pasteurella volantium $\mathrm{sp}$. nov. Int. J. Syst. Bacteriol. 35:5-9.

47. Namioka, S. 1978. Pasteurella multocida-biochemical characteristics and serotypes, p. 271-292. In T. Bergan and J. R. Norris (ed.), Methods in microbiology, vol. 10. Academic Press, Inc., London.

48. Newsom, I. E., and F. Cross. 1931. Some bipolar organisms found in pneumonia in sheep. J. Am. Vet. Med. Assoc. 80:711-719.

49. Pohl, S. 1981. DNA relatedness among members of Haemophilus, Pasteurella, and Actinobacillus, p. 245-253. In M. Kilian, W. Frederiksen, and E. L. Biberstein (ed.), Haemophilus, Pasteurella, and Actinobacillus. Academic Press, Inc., London.

50. PohI, S., H. U. Bertschinger, W. Frederiksen, and W. Mannheim. 1983. Transfer of Haemophilus pleuropneumoniae and the Pasteurella haemolytica-like organism causing porcine necrotic pleuropneumonia to the genus Actinobacillus (Acintobacilus pleuropneumoniae comb. nov.) on the basis of phenotypic and deoxyribonucleic acid relatedness. Int. J. Syst. Bacteriol. 33:510-514.

51. Regamey, R. 1939. Les infections humaines a $B$. bipolaris septicus (pasteurelloses). Éditions H. Huber, Bern.

52. Roberts, R. S. 1947. An immunological study of Pasteurella septica. J. Comp. Pathol. 57:261-278.

53. Rosenbusch, C. T., and I. A. Merchant. 1939. A study of the hemorrhagic septicemia pasteurella. J. Bacteriol. 37:69-89.

54. Skerman, V. B. D., V. McGowan, and P. H. A. Sneath (ed.). 1980. Approved lists of bacterial names. Int. J. Syst. Bacteriol. 30: $225-420$

55. Smith, J. E. 1959. Studies on Pasteurella septica. III. Strains from human beings. J. Comp. Pathol. 69:231-235.

56. Smith, J. E. 1961. The aerobic bacteria of the nose and tonsils of healthy dogs. J. Comp. Pathol. 71:428-433.

57. Smith, G. R. 1959. Isolation of two types of Pasteurella haemolytica from sheep. Nature (London) 183:1132-1133.
58. Sneath, P. H. A. 1982. Status of nomenclatural types in the Approved Lists of Bacterial Names. Int. J. Syst. Bacteriol. 32:459-460.

59. Sneath, P. H. A. 1983. Distortions of taxonomic structure from incomplete data on a restricted set of reference strains. J. Gen. Microbiol. 129:1045-1073.

60. Snieszko, S. F., G. L. Bullock, E. Hollis, and J. G. Boone. 1964. Pasteurella sp. from an epizootic of white perch (Roccus americanus) in Chesapeake Bay tidewater areas. J. Bacteriol. 88:1814-1815.

61. Snipes, K. P., and E. L. Biberstein. 1982. Pasteurella testudinis sp. nov.: a parasite of desert tortoises (Gopherus agassizi). Int. J. Syst. Bacteriol. 32:201-210.

62. Svoboda, K. H., S. Pohl, and W. Mannheim. 1981. Investigations on the phylogeny of Pasteurella multocida: DNA base sequence relatedness among the strains representing Carter's serogroups A through $\mathrm{E}$, and elimination of biovar 6 (so-called dog-type strains). Zentralbl. Bakteriol. Parasitenkd. Infektionskr. Hyg. Abt. 1 Orig. Reihe A 248:494-501.

63. Teissier, P., G. Gastinel, J. Reilly, and E. Rivalier. 1922. Contribution à l'étude des pasteurelloses à propos d'un cas de pasteurellose humaine. J. Physiol. Pathol. Gen. 20:221-225, 241-255.

64. Topley, W. W. C., and G. S. Wilson. 1929. The principles of bacteriology and immunity, 1st ed., vol. 1. E. Arnold, London.

65. Trevisan, V. 1885. Il fungo del cholera asiatico. Questioni risolte Atti Acad. Fisio. Med. Stat. Milano (Ser. 4) 3:78-91.

66. Trevisan, V.1887. Sul micrococco della rabbia e sulla possibilità di riconoscere durante il periodo d'incubazione, dall'esame del sangue della persona morsicata, se ha constratta l'infezione rabbica. Rend. R. Ist. Lomb. Sci. Let. Ser. II 20:88-105.

67. Ursing, J. 1981. Deoxyribonucleic acid hybridization studies of gas producing pasteurellae, p. 255-263. In M. Kilian, W. Frederiksen, and E. L. Biberstein (ed.), Haemophilus, Pasteurella, and Actinobacillus. Academic Press, Inc., London.

68. White, D. C. 1965 . Respiratory system in the hemin-requiring Haemophilus species. J. Bacteriol. 85:84-96.

69. Zablen, L. B., L. Bonen, R. Meyer, and C. R. Woese. 1975. The phylogenetic status of Pasteurella pestis. J. Mol. Evol. 4:347-358. 